\title{
Drosophila gustatory projections are segregated by taste modality and connectivity
}

Stefanie Engert ${ }^{1,2}$, Gabriella R. Sterne ${ }^{1}$, David T. Harris ${ }^{1,3}$, and Kristin Scott ${ }^{1, *}$

${ }^{1}$ University of California, Berkeley, United States

2Janelia Research Campus, Howard Hughes Medical Institute, United States

${ }^{3}$ current address: Calico Life Sciences, San Francisco, United States

${ }^{*}$ Corresponding author

\begin{abstract}
Gustatory sensory neurons detect caloric and harmful compounds in potential food and convey this information to the brain to inform feeding decisions. To examine the signals that gustatory neurons transmit and receive, we reconstructed gustatory axons and their synaptic sites in the adult Drosophila melanogaster brain, utilizing a whole-brain electron microscopy volume. We reconstructed 87 gustatory projections from the proboscis labellum in the right hemisphere and 57 in the left, representing the majority of labellar gustatory axons. Morphology- and connectivity-based clustering revealed six distinct clusters, likely representing neurons recognizing different taste modalities. Gustatory neurons contain a nearly equal number of interspersed pre-and post-synaptic sites, with extensive synaptic connectivity among gustatory axons. The vast majority of synaptic connections are between morphologically similar neurons, although connections also exist between distinct neuronal subpopulations. This study resolves the anatomy of labellar gustatory projections, reveals that gustatory projections are likely segregated based on taste modality, and uncovers synaptic connections that may alter the transmission of gustatory signals.
\end{abstract}

\section{Introduction}

All animals have specialized sensory neurons dedicated to the detection of the rich variety of chemicals in the environment that indicate the presence of food sources, 
predators and conspecifics. Gustatory sensory neurons have evolved to specialize in detecting food-associated chemicals and report the presence of caloric or potentially harmful compounds. Examining the activation and modulation of gustatory sensory neurons is essential as it places fundamental limits on the taste information that is funneled to the brain and integrated to form feeding decisions.

The Drosophila melanogaster gustatory system is an attractive model to examine the signals that gustatory neurons transmit and receive. Molecular genetic approaches coupled with physiology and behavior have established five different classes of gustatory receptor neurons (GRNs) in adult Drosophila that detect different taste modalities. One class, expressing members of the Gustatory Receptor (GR) family including Gr5a and Gr64f, detects sugars and elicits acceptance behavior (Dahanukar et al 2001, Dahanukar et al 2007, Thorne et al 2004, Wang et al 2004). A second class expressing different GRs including Gr66a detects bitter compounds and mediates rejection behavior (Thorne et al 2004, Wang et al 2004, Weiss et al 2011). A third class contains the ion channel PPK28 and detects water (Cameron et al 2010, Chen et al 2010). The fourth expresses the PPK23 ion channel and mediates detection of high salt concentrations, whereas the fifth expresses the Ir94e ionotropic receptor and detects low salt concentrations (Jaeger et al 2018). In addition to well-characterized gustatory neurons and a peripheral strategy for taste detection akin to mammals, the reduced number of neurons in the Drosophila nervous system and the availability of high-resolution electron microscopy brain volumes offer the opportunity to examine gustatory transmission with high resolution.

The cell bodies of gustatory neurons are housed in chemosensory bristles in peripheral tissues including the proboscis labellum, which is an external mouthparts organ that detects taste compounds prior to ingestion (Stocker 1994). Gustatory neurons from each labellum half send bilaterally symmetric axonal projections to the subesophageal zone (SEZ) of the fly brain via the labial nerves. Gustatory axons terminate in the medial SEZ in a region called the anterior central sensory center (ACSC) (Hartenstein et al 2018, Miyazaki \& Ito 2010, Thorne et al 2004, Wang et al 2004). Axons from bitter gustatory neurons send branches to the midline and form an interconnected medial ring whereas other gustatory axons remain ipsilateral and anterolateral to bitter projections. Although 
projections of different gustatory classes have been mapped using light level microscopy, the synaptic connectivity of gustatory axons in adult Drosophila is largely unexamined.

To explore the connectivity of GRNs and to lay the groundwork to study gustatory circuits, we used the recently available Full Adult Fly Brain Electron Microscopy dataset to fully reconstruct gustatory axons and their synaptic sites (Zheng et al 2018). We reconstructed 87 GRN axonal projections in the right hemisphere and 57 in the left, representing between $83-96 \%$ and $54-63 \%$ of the total expected, respectively. We used morphology- and connectivity-based clustering to identify six distinct clusters, likely representing neurons recognizing different taste modalities. By annotating chemical synapses, we observed that GRNs contain a nearly equal number of interspersed preand post-synaptic sites. Interestingly, many synaptic connections are among GRNs, with GRN-GRN connections yielding four distinct groups of connected neurons. Three of these groups segregate neurons based on taste modality, whereas one group contains neurons representing at least two modalities. We used calcium imaging approaches to test the function of GRN-GRN connectivity between taste modalities and observed neither crosscategory excitation nor inhibition. Additionally, we characterized the taste response profile of a second order neuron downstream of candidate sugar GRNs in the EM and found that it responds selectively to sucrose. Our study reveals extensive anatomical connectivity between gustatory sensory neurons, arguing for pre-synaptic processing of taste information prior to transmission to downstream circuits.

\section{Results}

\section{GRN axons contain pre-synaptic and post-synaptic sites}

To systematically characterize gustatory inputs and outputs, we manually traced gustatory axons in the Full Adult Fly Brain (FAFB) Electron Microscopy dataset, using the annotation platform CATMAID (Saalfeld et al 2009, Zheng et al 2018). GRNs from the proboscis labellum send axons through the labial nerve to the SEZ (Figure 1A). The labial nerve is a compound nerve, carrying sensory axons from the labellum, maxillary palp, and eye, as well as motor axons innervating proboscis musculature (Hampel et al 2020, Hartenstein et al 2018, Miyazaki \& Ito 2010, Nayak \& Singh 1983, Rajashekhar \& Singh 1994). Different sensory afferents occupy different domains in the SEZ, with labellar 
gustatory axons terminating in the anterior central sensory center (ACSC) (Hartenstein et al 2018, Miyazaki \& Ito 2010, Thorne et al 2004, Wang et al 2004). Therefore, to trace gustatory axons, we began by tracing neurites in the right labial nerve, readily identifiable in the EM dataset (Figure $1 \mathrm{~B}$ and $\mathrm{C}$ ), and selected fibers that terminated in the anterior central SEZ to trace to completion.

In tracing axons, we found that neurites with small to medium sized diameters in the dorsomedial labial nerve (Figure 1C) projected along a single neural tract (Figure 1D) to the anterior central region of the SEZ. This neural tract served as an additional site to select arbors for reconstruction. Individual fibers followed along the same tract and showed variation in terminal branching (Figure 1E). In total, we identified 87 axonal projections in the right hemisphere. Tracing from the left labial nerve and neural tract in the left hemisphere, we identified an additional 57 projections. There are 90-104 GRNs per labellum half (Jaeger et al 2018, Stocker 1994), arguing that we have identified 83$96 \%$ of the GRN fibers from the right labellum and $54-63 \%$ from the left. The projections from the left and right labial nerves are symmetric and converge in a dense web in the anterior central SEZ (Figure 1F). This arborization pattern recapitulates the labellar sensory projections of the ACSC, and we confirmed that the reconstructed neurites overlap with the known projection pattern of sugar and bitter sensory neuron in the standard fly brain space (Figure 1 - figure supplement 1) (Hartenstein et al 2018).

In addition to the skeleton reconstructions, we manually annotated pre- and postsynaptic sites. The presence of t-shaped structures characteristic of presynaptic release sites ('T bars'), synaptic vesicles, and a synaptic cleft were used to identify a synapse, consistent with previous studies (Zheng et al 2018). We found that synapses are sparse along the main neuronal tract and abundant at the terminal arborizations (Figure 1E). Interestingly, each GRN has a large number of pre- and post-synaptic sites intermixed along the arbors (Figure 1E and G-I). On average, a GRN contains 175 presynaptic sites and 168 postsynaptic sites, with individual GRNs showing wide variation in pre- and post- synapse number (Figure 1 - figure supplement 2). Our EM reconstructions reveal that GRNs represent a morphologically diverse population that not only relays taste information to the brain but also receives extensive synaptic inputs. 


\section{Different GRN classes can be identified by morphology and connectivity}

Drosophila GRNs comprise genetically defined, discrete populations that are specialized for the detection of specific taste modalities (Wang et al 2004, Cameron et al 2010, Jaeger et al 2018). As the EM dataset does not contain markers to distinguish between GRNs recognizing different taste modalities, we set out to identify subpopulations of reconstructed GRNs based on their anatomy and connectivity.

First, we used the NBLAST algorithm to perform automated clustering of GRN axons to define different subpopulations based on their morphology (Costa et al 2016). Each traced skeleton on the right was registered to a standard template brain (Bogovic et al 2018). All 144 reconstructed GRNs were compared pairwise in an all-by-all matrix. For each pair, an NBLAST neuronal similarity score was computed based on the position and geometry of the query and target GRN. We then used Ward's method to hierarchically cluster GRNs into groups (Ward 1963). We chose seven groups as the number that minimizes within-cluster variance (Figure 2 - figure supplement 1) (Braun et al 2010). This anatomical clustering revealed morphologically similar groups on the two hemispheres

We found, however, that this purely anatomical approach did not align well with connectivity patterns observed between GRNs (Figure 1 - figure supplement 2). To capitalize on the phenomenon of GRN-GRN connectivity in our clustering, we confined the analysis to GRNs in the right hemisphere because tracing is near completion and consequently represents a fuller connectivity pattern. In addition to morphology, connectivity data comprised of all synapses between each pair of neurons was included in our clustering analysis. Using this combination of morphological and connectivity-based clustering, we found six GRN clusters (Figure 2A).

These six GRN clusters are morphologically distinct, occupying discrete zones in the SEZ and sharing anatomically similar terminal branches (Figure $2 \mathrm{~B}$ ). To evaluate whether the different clusters represent GRNs detecting different taste modalities, we examined the number of GRNs in each group and their anatomy. Based on previous studies (Jaeger et al 2018), there are 20 bitter-sensing GRNs, 9 low salt GRNs, 15 high salt GRNs, 31 sugar GRNs, and 15 water GRNs per labellum half. In addition, bitter GRN axons cross the midline whereas other GRN axons do not. Cluster 1 and 2 cross the midline and comprise 19 GRNs, suggesting that they represent bitter GRNs (Figure 2C 
and D) (Wang et al 2004, Thorne et al 2004). Group 3 contains 12 GRNs, with projections that resemble low salt GRNs (Figure 2E) (Jaeger et al 2018). Group 4 contains 17 GRNs that we hypothesize are water GRNs based on anatomy (Figure 2F) (Cameron et al 2010, Chen et al 2010). Group 5 has 16 GRNs and most resembles the projection patterns of sugar neurons (Figure 2G) (Jaeger et al 2018). Group 6 contains 23 GRNs and may correspond to high salt GRNs (Figure 2H) (Wang et al 2004, Thorne et al 2004). We emphasize GRNs in cluster 4-6 are anatomically very similar, so that identity assignments are tentative.

\section{GRNs are highly interconnected via chemical synapses}

During our reconstruction of GRNs in the EM dataset, we noticed that GRNs are both pre- and post-synaptic to other GRNs. We determined that each GRN receives between $2 \%$ and $66 \%$ (average $=39 \%$ ) of its synaptic input from other GRNs (Figure 3A). The number of synapses between GRNs suggests that communication between sensory neurons may directly regulate sensory output. To gain further insight into GRN-GRN communication, we focused on connections comprised of five or more synapses, consistent with previous studies (Buhmann et al 2021, Li et al 2020a, Takemura et al 2013, Takemura et al 2015).

To examine whether connections exist exclusively between neurons of the same anatomy- and connectivity- based cluster, potentially representing the same taste modality, or between multiple clusters, we plotted the connections between all GRNs in a matrix (Figure 3B). We found that GRNs are connected in four distinct groups, with extensive synapses between individual GRNs. One group of interconnected neurons contains all neurons of clusters 1 and 2, likely representing bitter GRNs. A second group contains neurons of cluster 3, likely representing low-salt GRNs. A third connectivity group is comprised of cluster 5, likely representing high-salt GRNs. The fourth connectivity group contains mostly neurons of clusters 4 and 6 , which we postulate are water and sugar GRNs. At 42 neurons, this anatomically mixed connectivity group is too large to correspond to neurons of a single taste modality. Thus, while three groups of synaptically connected clusters correspond to anatomical classes, one group contains neurons of multiple anatomical classes, suggesting pre-synaptic communication both 
within neurons representing a single taste modality and between neurons of different modalities.

Comparing the synaptic connectivity within and between anatomy- and connectivity-based clusters reveals that the vast majority of synapses are between neurons of the same cluster (Figure 3C). For example, cluster 4 neurons receive 1213 synapses from other cluster 4 neurons and 85 from other neurons. The large number of synapses between neurons belonging to the same anatomical class suggests that communication between GRNs of the same taste modality is predominant. However, there are also a small number of synapses between GRNs detecting different modalities.

Overall, we found large numbers of chemical synapses between GRNs. Connectivity between GRNs of the same anatomical cluster could provide a mechanism to amplify signals of the same taste modality. In addition, the connectivity between GRNs of different anatomical clusters could serve to integrate taste information from different modalities before transmission to downstream circuitry.

\section{Interactions between sugar and water GRNs are not observed by calcium or voltage imaging}

To examine whether the small number of connections between GRNs of different taste modalities results in cross-activation of GRNs detecting different primary tastant classes, we explored if activation of one GRN class results in propagation of activity to other GRN classes. As the connectivity data suggests that sugar and water GRNs are weakly connected, we wondered if appetitive GRNs might be interconnected to amplify appetitive signals to downstream feeding circuits. To test for interactions between appetitive GRNs, we undertook calcium and voltage imaging studies in which we monitored the response of a GRN class upon activation of other GRN classes.

We expressed the calcium indicator GCaMP6s in genetically defined sugar, water or bitter sensitive GRNs to monitor excitatory responses upon artificial activation of different GRN classes. To ensure robust and specific activation of GRNs, we expressed the mammalian ATP receptor P2X2 in sugar, water or bitter GRNs, and activated the GRNs with an ATP solution presented to the fly proboscis while imaging gustatory projections in the brain (Yao et al 2012, Harris et al 2015). Expressing both P2X2 and 
GCaMP6s in sugar, water or bitter GRNs elicited strong excitation upon ATP presentation (Figure 4A-B and G-H and Figure 4 - figure supplement 1-3 C-D). As bitter cells are synaptically connected to each other but not to sugar or water cells, we hypothesized that they would not be activated by sugar or water GRN activation. Consistent with the EM connectivity, activation of sugar or water GRNs did not activate bitter cells, nor did bitter cell activation elicit responses in sugar or water axons (Figure 4 - figure supplement 1E$\mathrm{H}$; Figure 4 - figure supplement 2E-F; Figure 4 - figure supplement 3G-H). In contrast, the EM connectivity indicates interactions between sugar and water GRNs. However, we did not observe responses in sugar GRNs upon water GRN activation (Figure 4C-D; Figure 4 - figure supplement 2IJ) or responses in water GRNs upon sugar GRN activation (Figure $4 \mathrm{I}$ and J; Figure 4 - figure supplement $3 \mathrm{E}$ and F). To examine whether interactions between modalities are modulated by the feeding state of the fly, we performed the activation and imaging experiments in both fed and starved flies (Figure 4 - figure supplement 1-6). These experiments did not reveal feeding state dependent interactions between GRN populations.

We hypothesized that interactions between sugar and water GRNs might be inhibitory, providing a mechanism to weight different appetitive taste inputs. To examine this, we expressed the voltage indicator ArcLight, which preferentially reports hyperpolarization, in sugar GRNs while activating water GRNs via P2X2 and vice versa. These experiments revealed no change in voltage in one appetitive gustatory class upon activation of the other (Figure 4E-F and K-L: Figure 4 - figure supplement 7). Overall, despite the potential for crosstalk between different modalities revealed by EM, we observed no communication between appetitive GRNs by calcium or voltage imaging of gustatory axons.

\section{A second-order taste neuron responds selectively to sucrose and causes proboscis extension}

To further test the potential interaction between sugar and water sensory neurons and to validate the identification of GRN subtypes in the EM dataset, we sought to trace a second-order gustatory neuron and test if it responds to both sugar and water taste detection. By tracing from a cluster 4 GRN contained within connectivity group 4 (Skeleton 
ID 7349219), we identified a second-order neuron predominantly connected to cluster 5 GRNs with comparatively few inputs from clusters 4 and 6 (Figure 5A and E-F). We identified a Gal4 line containing this second order neuron (Figure 5C, Figure 5 - figure supplement 1), which we named Famine (FMIn). FMIn extensively overlaps with candidate sugar sensing GRNs in the EM dataset (Figure 5B), forming 331 synapses (Figure 5E and F). This anatomical overlap is also observable by colabeling studies of sugar GRNs and FMIn single-cell mosaics (Figure 5D)(Gordon \& Scott 2009).

We tested the function of FMIn using calcium imaging and behavioral approaches. Monitoring the response of FMIn to taste compounds delivered to the fly proboscis revealed that FMIn responded selectively to sucrose but not to water or bitter compounds in food-deprived flies, demonstrating that FMIn is a second-order sugar-sensing gustatory neuron (Figure 5G and $\mathrm{H}$ ). The calcium imaging studies also argue that excitatory interactions between sugar and water GRNs are not readily apparent in second-order neuron activity. To test the behavioral function of FMIn, we activated it using specific expression of a red shifted light-gated cation channel, ReaChR, and found that this elicited proboscis extension, a behavior produced by activation of appetitive GRNs (Figure 5I). We used mosaic approaches to restrict expression of ReaChR and found that the strength of the response was dependent on whether ReaChR was expressed in only one or both FMIns. These studies demonstrate the sugar selectivity of a second-order gustatory neuron and validate the EM classification of sugar-sensing GRNs.

\section{Discussion}

In this study, we characterized different classes of gustatory projections and their interconnectivity by high-resolution EM reconstruction. We identified different projection patterns corresponding to gustatory neurons recognizing different taste modalities. The extensive connections between GRNs of the same taste modality as well as the possible interactions between GRNs of two modalities provide anatomical evidence of pre-synaptic processing of gustatory information.

An emerging theme stemming from EM reconstructions of Drosophila sensory systems is that sensory neurons of the same subclass are synaptically connected. In general, different sensory neuron subclasses have spatially segregated axonal termini in 
the brain, thereby constraining the potential for connectivity. In the adult olfactory system, approximately $40 \%$ of the input onto olfactory receptor neurons (ORNs) comes from other ORNs projecting to the same olfactory glomerulus (Horne et al 2018, Schlegel et al 2021, Tobin et al 2017). Similarly, mechanosensory projections from the Johnston's Organ of the same submodality are anatomically segregated and synaptically connected (Hampel et al 2020). In Drosophila larvae, $25 \%$ of gustatory neuron inputs are from other GRNs, although functional classes were not resolved (Miroschnikow et al 2018). In the adult Drosophila gustatory system, we also find that GRNs are interconnected, with approximately $39 \%$ of GRN input coming from other GRNs. Consistent with other classes of sensory projections, we find that gustatory projections are largely segregated based on taste modality and form connected groups. A general function of sensory-sensory connections seen across sensory modalities may be to enhance weak signals or to increase dynamic range.

By clustering neurons based on anatomy and connectivity, we were able to resolve different GRN categories. The distinct morphologies of bitter neurons and low saltsensing neurons, known from immunohistochemistry, are recapitulated in the projection patterns of clusters 1 and 2 and 3 , enabling high-confidence identification. The projections of high salt, sugar and water-sensing neurons are ipsilateral, with similarities in their terminal arborizations (Jaeger et al 2018, Wang et al 2004). Nevertheless, comparisons between EM and light-level projections argue that these taste categories are also resolved into different, identifiable clusters. However, as these categories are based on anatomical comparisons alone, they remain tentative until further examination of taste response profiles of connected second-order neurons, now identifiable by EM.

Whereas clustering identified six different clusters, examining GRN-GRN connectivity revealed four synaptically connected groups. Three groups connect neurons of the same taste modality, whereas the fourth group likely contains both water and sugar neurons. While it is tempting to speculate that interactions between appetitive tastes may amplify or filter activation of feeding circuits, we were unable to identify cross-activation between sugar and water GRNs by calcium or voltage imaging. We also found no evidence for cross-activation of sugar and water sensory neurons when monitoring activity of a second-order gustatory neuron. It is possible that these interactions are 
dependent on the feeding state of the animal or act on a timeframe not examined in this study. Alternatively, activation may be highly localized to the synaptic terminal and not resolved in these studies. Finally, the number of synapses is approximately 15-fold fewer between anatomical classes than within classes, suggesting that the small number of synapses may not be relevant for taste processing.

Overall, this study resolves the majority of labellar gustatory projections and their synaptic connections, revealing that gustatory projections are segregated based on taste modality and synaptically connected. The identification of GRNs detecting different taste modalities now provides an inroad to enable the examination of the downstream circuits that integrate taste information and guide feeding decisions.

\section{Materials and Methods \\ Experimental Animals}

Experimental animals were maintained on standard agar/molasses/cornmeal medium at $25^{\circ} \mathrm{C}$. For imaging experiments requiring food-deprived animals, flies were placed in vials containing wet kimwipes for 23-26 hours immediately prior to the experiment. For behavior experiments, flies were placed on food supplemented with $400 \mu \mathrm{M}$ all trans retinal for 24 hours prior to the experiment.

Transgenic animals used were: Gr64f-LexA (Miyamoto et al 2012); Gr64f-Gal4 (Kwon et al 2011); Gr66a-LexA; ppk28-LexA; UAS-CD::tdTomato; LexAop-Gal80 (Thistle et al 2012); Gr66a-Gal4 (Scott et al 2001); ppk28-Gal4 (Cameron et al 2010); LexAopGCaMP6s (Chen et al 2013); UAS-GCaMP7s (Dana et al 2019), UAS-ArcLight (Cao et al 2013); UAS-P2X2 (Lima \& Miesenbock 2005); GmR81E10-Gal4 (Jennet et al 2012); Flippase 1026a (Huang et al 2014); UAS>mcherry::stop>ReaChR::Citrine (Inagaki et al 2013); UAS-CD8::GFP (Lee \& Luo, 1999); Gr5a-LexA, tub>Gal80> (Gordon \& Scott 2009); LexAop-myr::tdTomato (Nern et al 2011).

\section{EM reconstruction}

Neuron skeletons were reconstructed in a serial sectioned transmission electron microscopy dataset of the whole fly brain (Zheng et al 2018) using the annotation software CATMAID (Saalfeld et al 2009). GRN projections were identified based on their extension into the labial nerve and localization to characteristic neural tracts in the SEZ. To identify 
second order neurons, random presynapses of skeleton 7349219 were chosen using the reconstruction sampler function of CATMAID and downstream partners were reconstructed. Skeletons were traced to completion either entirely manually or using a combination of an automated segmentation ( $\mathrm{Li}$ et al 2020b) and manual tracing as previously described (Hampel et al 2020). Chemical synapses were annotated manually using criteria previously described (Zheng et al 2018). Skeletons were reviewed by a second specialist, so that the final reconstruction presents the consensus assessment of at least two specialists. Skeletons were exported from CATMAID as swc files for further analysis, and images of skeletons were exported directly from CATMAID. FAFB neuronal reconstructions will be available from Virtual Fly Brain (https://fafb.catmaid.virtualflybrain.org/).

\section{Clustering of GRNs}

GRNs were hierarchically clustered based on morphology and connectivity using NBLAST and synapse counts. First, GRN skeletons traced in FAFB were registered to the JRC2018U template (Bogovic et al 2018) and compared in an all-by-all fashion with NBLAST (Costa et al. 2016). NBLAST analysis was carried out with the natverse toolkit in R (Bates et al. 2020; R Development Core Team, https://www.r-project.org/). The resulting matrix of NBLAST scores was merged with a second matrix containing all-by-all synaptic connectivity counts for the same GRNs. The resulting merged matrix was absolute maximum scaled such that all values fall within the range of 0 and 1 . The merged, scaled matrix was hierarchically clustered using Ward's method (Ward 1963) in Python (Python Software Foundation, https://www.python.org/) with SciPy (Virtanen et al 2020). Five groups were chosen based on prior knowledge of taste modalities, as GRNs for sugar, water, bitter, high salt, and low salt are known to be functionally, morphologically, and genetically distinct.

Connectivity data of GRNs was exported from CATMAID for further analysis and connectivity diagrams were generated using CytoScape (Shannon et al 2003).

\section{Calcium and Voltage Imaging Preparation}

For imaging studies of GRNs, mated females, 10 to 21 days post eclosion, were dissected as previously described (Harris et al 2015), so that the brain was submerged in artificial hemolymph (AHL) while the proboscis was kept dry and accessible for taste 
stimulation. To avoid occlusion of taste projections in the SEZ, the esophagus was cut. The front legs were removed for tastant delivery to the proboscis. AHL osmolality was assessed as previously described (Jourjine et al 2016) and adjusted according to the feeding status of the animal. In fed flies, AHL of 250 mOsmo was used (Wang et al 2003). The AHL used for starved flies was diluted until the osmolality was $180 \mathrm{mOsmo}$, consistent with measurements of the hemolymph osmolality in food deprived flies (Jourjine et al 2016).

For imaging studies of FMIn, mated females, 3 to 4 days post eclosion, were deprived of food for 18 to 24 hours prior to imaging in an empty vial with a moist kimwipe. Flies were dissected as described above in $\sim 180 \mathrm{mOsmo} A H L$.

\section{Calcium Imaging}

Calcium transients reported by GCaMP6s and GCaMP7s were imaged on a 3i spinning disc confocal microscope with a piezo drive and a 20x water immersion objective $(\mathrm{NA}=1)$. For our studies of GRNs, stacks of $14 \mathrm{z}$ sections, spaced 1.5 microns apart, were captured with a 488nm laser for 45 consecutive timepoints with an imaging speed of $\sim 0.3$ $\mathrm{Hz}$ and an optical zoom of 2.0. For better signal detection, signals were binned $8 \times 8$, except for Gr64f projections, which underwent 4x4 binning. For our studies of FMIn, stacks of $20 \mathrm{z}$ sections spaced 1.5 microns apart were captured with an $488 \mathrm{~nm}$ laser for 25 consecutive timepoints with an imaging speed of $\sim 0.3 \mathrm{~Hz}$ and an optical zoom of 1.6.

\section{Voltage Imaging}

Voltage responses reported by ArcLight were imaged similarly to the calcium imaging outlined above. To increase the imaging speed, the number of $z$ planes was reduced to 10 , and the exposure time was decreased from $100 \mathrm{~ms}$ to $75 \mathrm{~ms}$, resulting in an imaging speed of $\sim 0.7 \mathrm{~Hz}$. To maintain a time course comparable to that of the calcium imaging experiments of GRNs, the number of timepoints was increased to 90 . Signals were binned $8 \times 8$ in each experiment.

\section{Taste stimulations}

Taste stimuli were delivered to the proboscis via a glass capillary as previously described (Harris et al 2015). For GRN studies, each fly was subjected to three consecutive imaging sessions, each consisting of a taste stimulation at time point 15, 25 and 35 (corresponding to $30,50.5,71.5 \mathrm{sec}$ ). During the first imaging session, the fly was 
presented with a $20 \%$ polyethylene glycol (PEG, average molecular weight $3350 \mathrm{~g} / \mathrm{mol}$ ) solution, acting as a negative control. PEG was used in all solutions except water solutions, as this PEG concentration inhibits activation of water GRNs (Cameron et al 2010). This was followed in the second session with stimulations with 100mM ATP in $20 \%$ PEG. In the last imaging session, each fly was presented with a tastant acting as a positive control in 20\% PEG (Gr64f: 1M sucrose; Gr66a: 100mM caffeine, 10mM denatonium benzoate; ppk28: $\mathrm{H} 2 \mathrm{O}$; ppk23: $1 \mathrm{M} \mathrm{KCl}$ in 20\% PEG).

For FMIn studies, flies were imaged for in three consecutive imaging sessions with tastant delivery at time point 15, corresponding to $\sim 2 \mathrm{sec}$. For the first of these sessions, $\mathrm{H} 2 \mathrm{O}$ was the tastant, for the second $100 \mathrm{mM}$ caffeine and $10 \mathrm{mM}$ denatonium benzoate in $20 \%$ PEG, and for the third $1 \mathrm{M}$ Sucrose in $20 \%$ PEG.

\section{Imaging Analysis}

Image analysis was performed in FIJI (Schindelin et al 2012). Z stacks for each time point were converted into $\max z$ projections for further analysis. After combining these images into an image stack, they were aligned using the StackReg plugin in FIJI to correct for movement in the xy plane (Thevenaz et al 1998).

For our exploration of interactions between GRN subtypes, one ROI was selected encompassing the central arborization of the taste projection in the left or right hemisphere of the SEZ in each fly. Whether the projection in the left or right hemisphere was chosen depended on the strength of their visually gauged response to the positive control. The exception were Gr66a projections, in which the entire central projection served as ROI. If projections did not respond strongly to at least two of the three presentations of the positive control, the fly was excluded from further analysis. If projections responded to two or more presentations of the negative control, the fly was excluded from further analysis. A large ROI containing no GCaMP signal was chosen in the lateral SEZ to determine background fluorescence.

In calcium imaging experiments, the first five time points of each imaging session were discarded, leaving 40 time points for analysis with taste stimulations at time points 10,20 and 30 . The average fluorescence intensity of the background ROI was subtracted at each time point from that of the taste projection ROI. F0 was then defined as the average fluorescence intensity of the taste projection ROI post background subtraction of 
the first five time points. $\Delta \mathrm{F} / \mathrm{F}(\%)$ was calculated as $100 \%{ }^{*}(\mathrm{~F}(\mathrm{t})-\mathrm{F} 0) / \mathrm{F} 0$. Voltage imaging experiments were analyzed similarly, with ten initial time points discarded for a total of 80 time point in the analysis and tastant presentations at time points 20, 40 and 60.

To study the taste responses in FMIn, an ROI encompassing the cell body of FMIn was selected. As the cell bodies of FMIn are commonly located extremely peripherally in the neuropil at or in the labial nerve, we chose to forgo background subtraction. Further image analysis was preformed like that of the calcium imaging experiments performed on GRNs outlined above.

\section{Quantification of Calcium and Voltage Imaging}

Graphs were generated in GraphPad Prism. To calculate the max $\Delta F / F(\%)$ of GCaMP responses, the $\Delta \mathrm{F} / \mathrm{F}(\%)$ of the three time points centered on the peak $\Delta \mathrm{F} / \mathrm{F}(\%)$ after the first stimulus response were averaged. The average $\Delta F / F(\%)$ of the three time points immediately preceding the stimulus onset were then subtracted to account for changing baselines during imaging. Arclight data was similarly analyzed, except that five timepoints centered on the peak $\Delta F / F(\%)$ and five time points prior to stimulus onset were considered. Statistical tests were performed in Prism as indicated in figures.

\section{Immunohistochemistry}

To visualize the expression pattern of Gmr81E10-Gal4 and the overlap of FMIn and Gr5a, mated female flies, 10 to 14 days post eclosion, were dissected in PBS. Fixation in 4\% paraformaldehyde and antibody staining was performed as previously described (Wang et al 2004). The primary antibodies used were mouse anti Brp (1:500), chicken anti GFP (Co-label only; 1:1000), rabbit anti GFP (Gal4 expression pattern only; 1:1000) and rabbit anti dsRed (Co-label only; 1:1000). The secondary antibodies used were goat anti rabbit AlexaFluor 488 (Gal4 Expression pattern only; 1:100), goat anti chicken AlexaFluor 488 (Co-label only; 1:1000), goat anti rabbit AlexaFluor 568 (Co-label only; $1: 1000)$, and goat anti mouse AlexaFluor 647 (1:100). All images were acquired on a Zeiss Axioscope upright confocal microscope, and their brightness and contrast were manually adjusted in FIJI.

Dissection and staining of GR64f and Gr66a projections were carried out by FlyLight according to the FlyLight 'IHC-Polarity Sequential Case 5' protocol (https://www.janelia.org/project-team/flylight/protocols). Samples were imaged on an 
LSM710 confocal microscope (Zeiss) with a Plan-Apochromat 20×/0.8 M27 objective. Images were registered using CMTK (https://www.nitrc.org/projects/cmtk) and manually segmented with VVDviewer (https://github.com/takashi310/VVD Viewer; Otsuna et al., 2018 ) in order to remove any non-specific background.

\section{PER Behavior}

Mated female flies, 3 to 10 days post eclosion, were briefly anesthetized with $\mathrm{CO} 2$ and glued to a microscope slide with a small drop of nail polish. They were allowed to recover for $\sim 2$ hours in a dark empty pipette tip box with a moist kimwipe to prevent desiccation. Their light response was examined under a dissection microscope by observing their behavior for several seconds in ambient light before turning on the microscope's light source aimed directly at the fly. Only if the fly fully extended its proboscis was it classified as a 'responder'. Flies extending their proboscis in ambient light were excluded from the analysis. Behavior was recorded using a small digital camera. Following the behavior experiments, flies were individually removed from the microscope slide and their brains dissected in PBS. For each fly, the expression pattern of UAS-ReaChR::Citrine was assessed using a fluorescence microscope. Flies in which ReaChR was expressed in cells other than FMIn were excluded from analysis.

\section{Acknowledgements}

We thank Lori Horhor, Jolie Huang, Neil Ming, and Parisa Vaziri for EM tracing contributions. This work was supported by NIH R01DC013280 (K.S.) and $\mathrm{NIH}$ F32DK117671 (G.S.). FAFB was used for EM reconstructions (Zheng 2018). We thank John Bogovic for registration of EM skeletons in the $2018 \mathrm{U}$ template. Neuronal reconstruction for this project took place in a collaborative CATMAID environment in which 27 labs are participating to build connectomes for specific circuits. Development and administration of the FAFB tracing environment and analysis tools were funded in part by National Institutes of Health BRAIN Initiative grant 1RF1MH120679-01 to Davi Bock and Greg Jefferis, with software development effort and administrative support provided by Tom Kazimiers (Kazmos $\mathrm{GmbH}$ ) and Eric Perlman (Yikes LLC). We thank Peter Li for sharing his automatic segmentation (Li et al 2019). 
bioRxiv preprint doi: https://doi.org/10.1101/2021.12.08.471796; this version posted December 9,2021. The copyright holder for this preprint (which was not certified by peer review) is the author/funder, who has granted bioRxiv a license to display the preprint in perpetuity. It is made available under aCC-BY-NC-ND 4.0 International license.

\section{Figure 1}
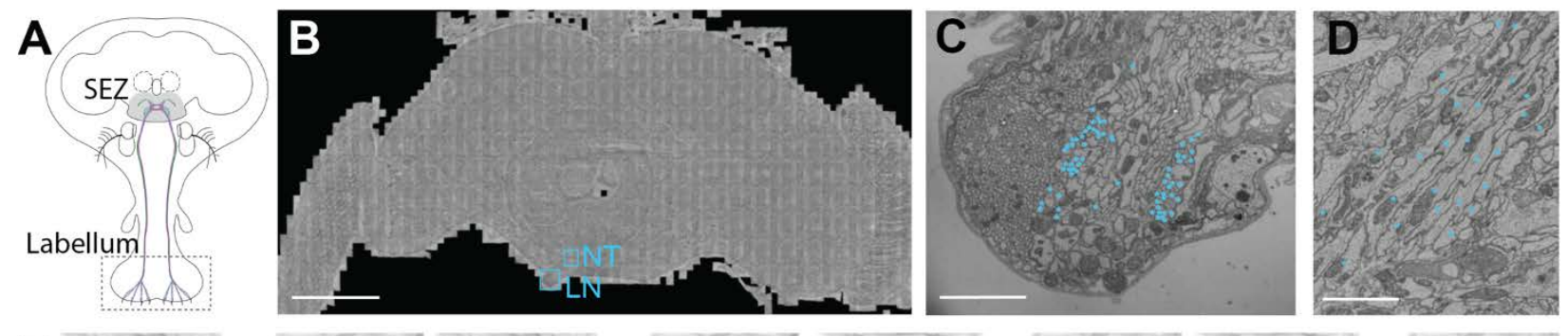

E
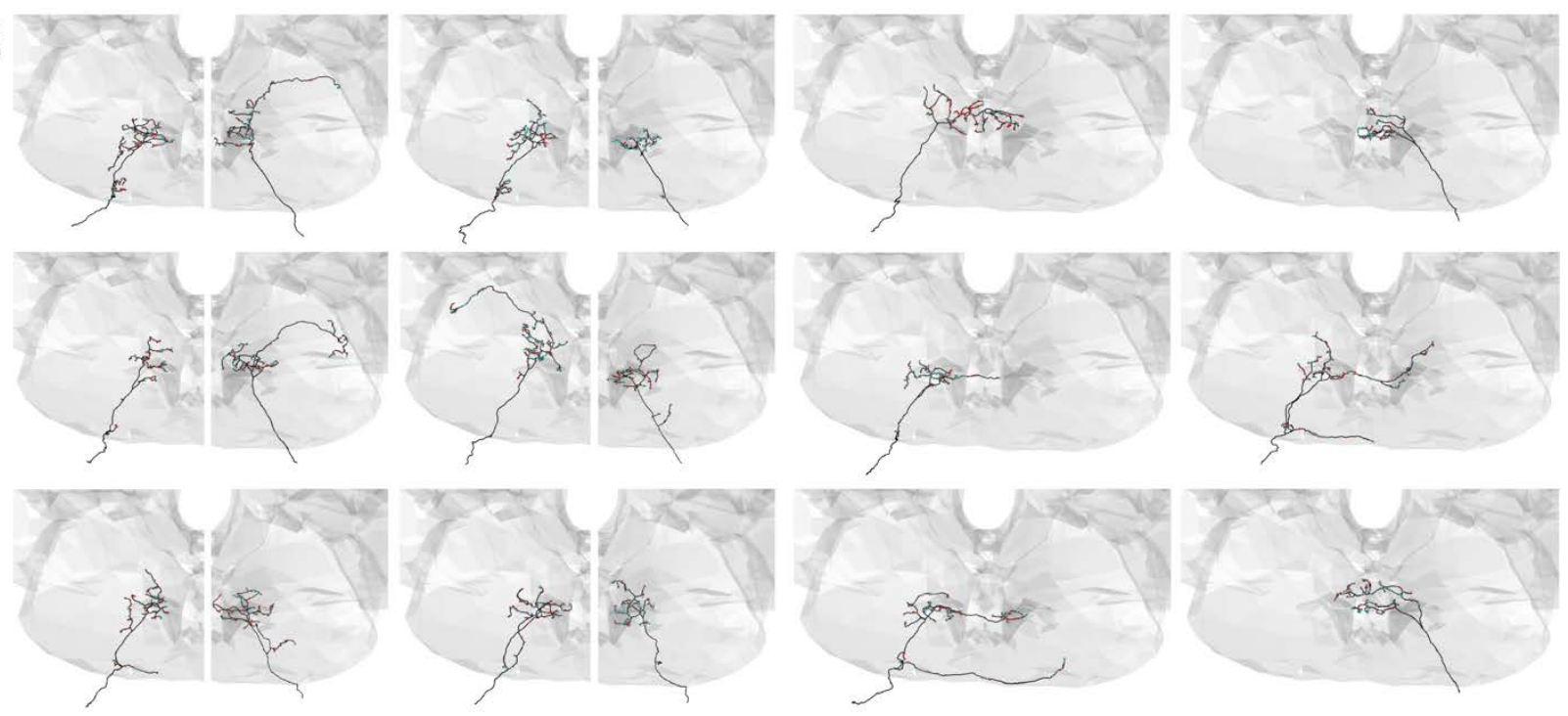

$\mathbf{F}$
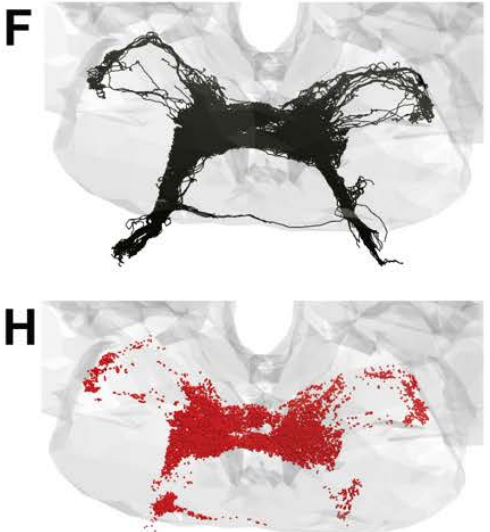
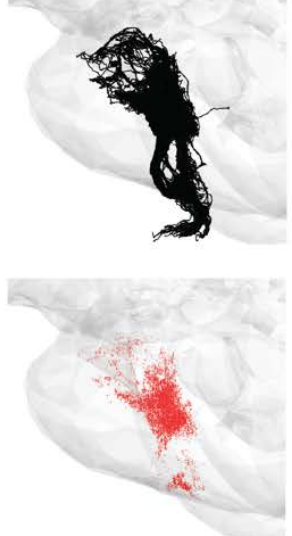
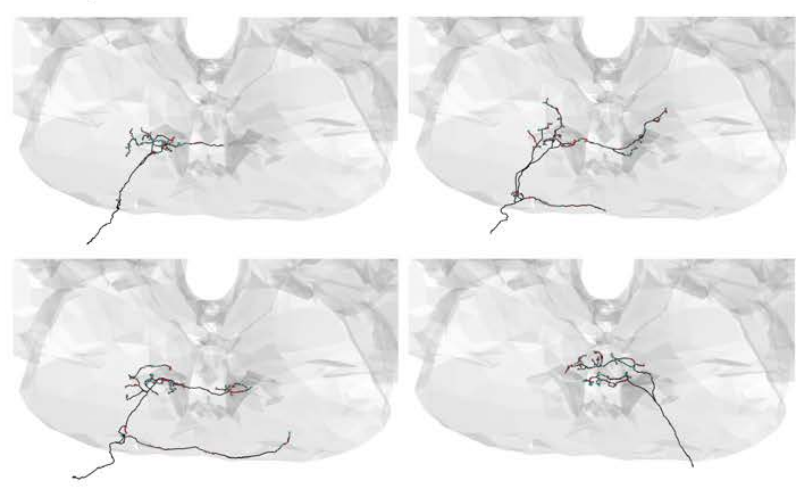

G
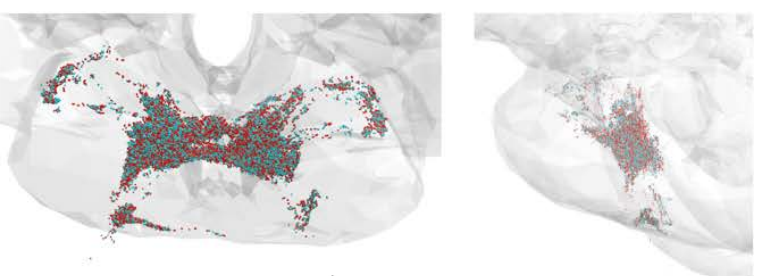

I
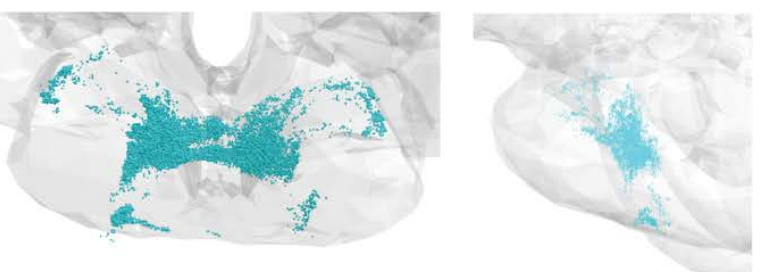
Figure 1. EM-based reconstructions of GRNs and synaptic sites. (A) Schematic showing GRNs in the proboscis labellum and their projections in the SEZ. (B) Location of the labial nerve (LN) and neural tract (NT) containing GRNs of the right hemisphere in the FAFB dataset (Z slice 3320, scale bar $=100 \mu \mathrm{M}$ ). (C) Cross-section of the labial nerve with traced GRNs indicated by asterisks (Z slice 3320 , scale bar $=5 \mu \mathrm{M}$ ). (D) Neural tract with traced GRNs indicated by asterisks (Z slice 2770 , scale bar $=5 \mu \mathrm{M}$ ). (E) Examples of reconstructed GRNs with presynaptic (red) and postsynaptic (blue) sites. (F-I) Frontal and sagittal view of all reconstructed GRN axons (F), all presynaptic (red) and postsynaptic (blue) sites $(\mathrm{G})$, presynaptic sites alone $(\mathrm{H})$, and postsynaptic sites alone (I). 

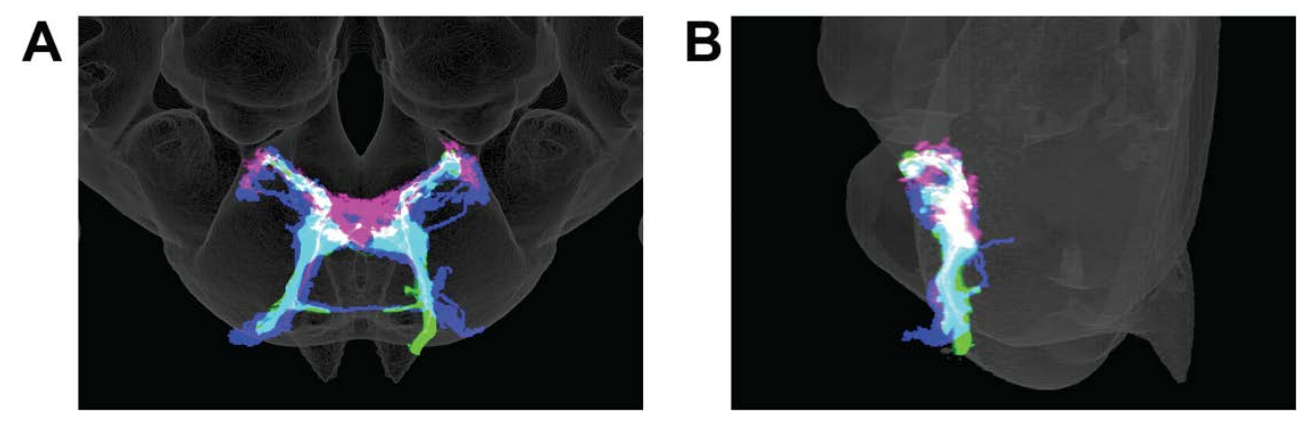

Figure 1 - figure supplement 1. Reconstructed GRN skeletons overlap with the projection patterns of bitter and sugar GRNs in the template brain space. (A) Frontal view of reconstructed GRNs (dark blue), sugar GRNs (green), and bitter GRNs (magenta) in the $2018 \mathrm{U}$ template brain. (B) Sagittal view of the neurons in A. 


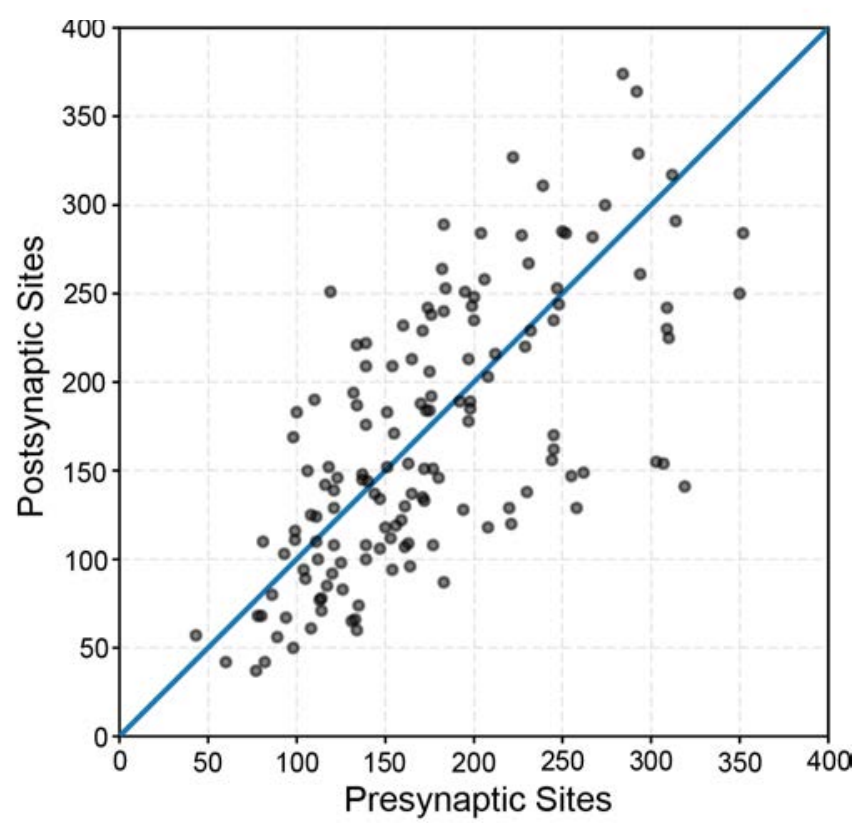

Figure 1 - figure supplement 2. GRNs contain similar numbers of pre- and postsynaptic sites. Plot of pre- and post-synaptic sites for individual GRNs, denoted by grey circles. Diagonal line indicates one-to-one relationship of pre- and post-synaptic sites. 


\section{Figure 2}

A
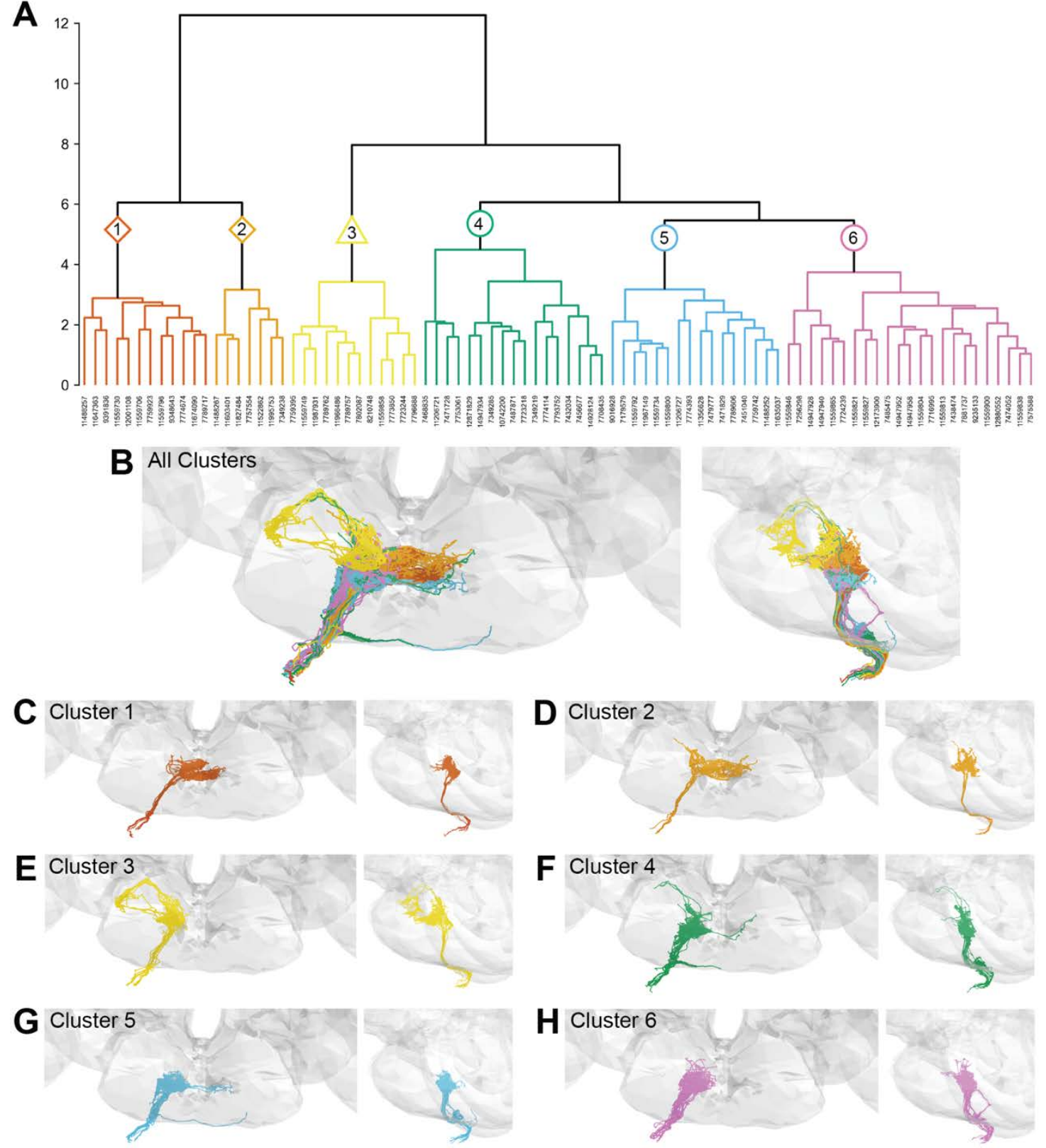

Figure 2. Morphology- and connectivity-based clustering generates distinct groups of GRNs. (A) Tree denoting relative similarity of GRNs based on morphology and connectivity of GRNs in the right hemisphere. (B) Frontal and sagittal view of all GRN clusters, colored according to A. (C) Frontal and sagittal view of cluster $1 \mathrm{GRNs.}$ (D) Frontal and sagittal view of cluster 2 GRNs. (E) Frontal and sagittal view of cluster 3 GRNs. (F) Frontal and sagittal view of cluster 4 GRNs. (G) Frontal and sagittal view of cluster 5 GRNs. (H) Frontal and sagittal view of cluster 6 GR 


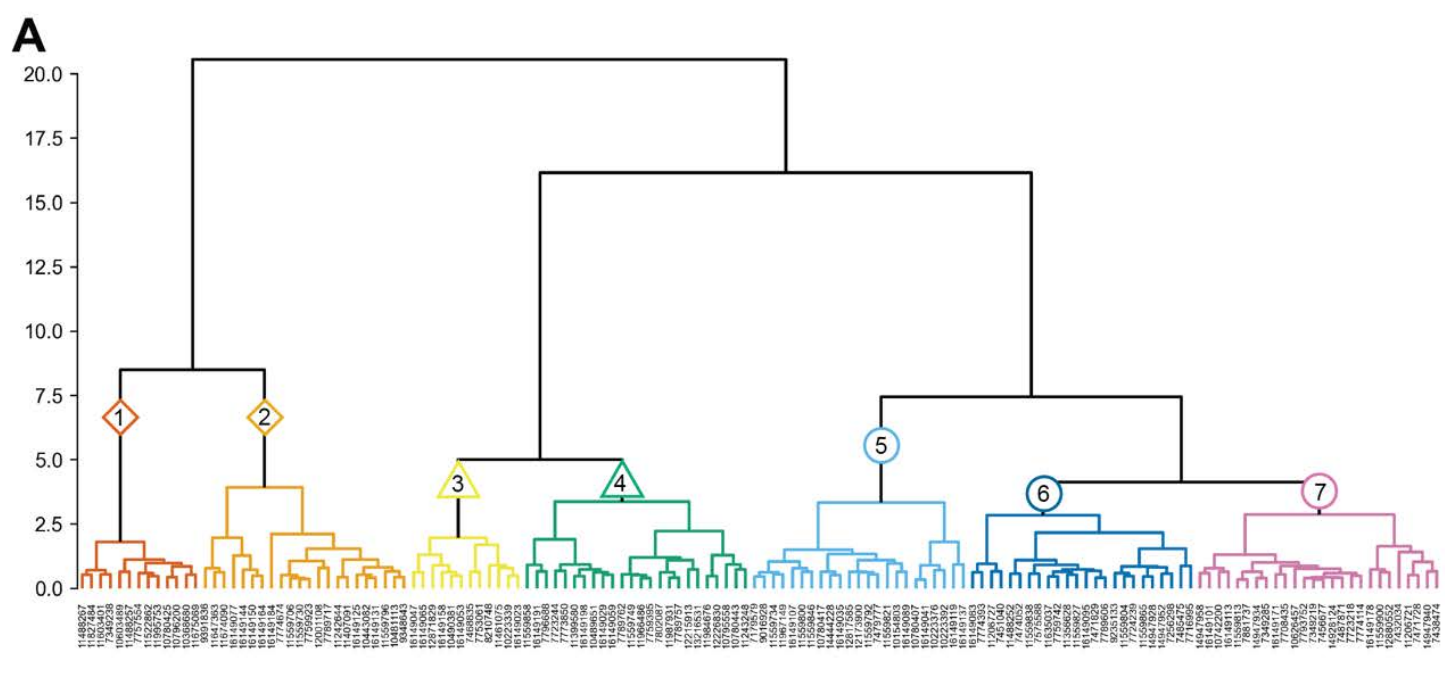

B All Clusters

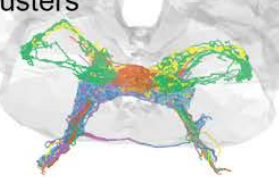

D Cluster 2

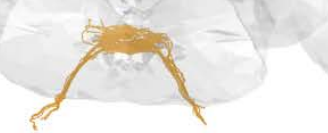

F Cluster 4

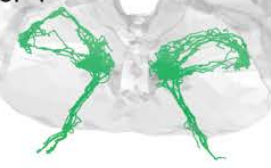

H Cluster 6

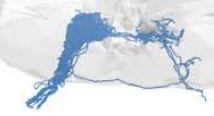

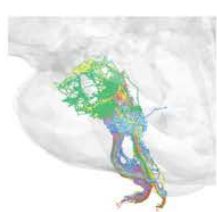
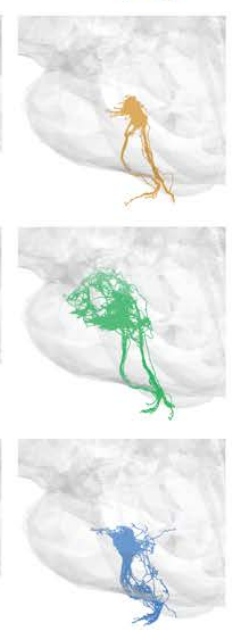

C Cluster 1

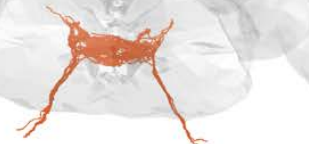

E Cluster 3

G Cluster 5

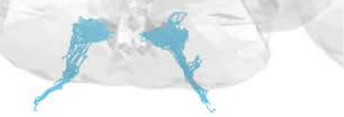

Cluster 7

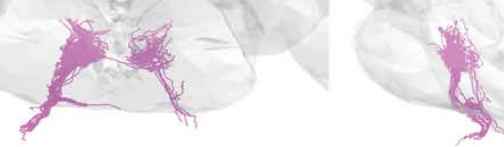

Figure 2 - figure supplement 1. Morphology-based clustering generates distinct groups of GRNs. (A) Tree denoting relative similarity of all GRNs based on morphology alone. (B) Frontal and sagittal view of all GRN clusters, colored according to A. (C) Frontal and sagittal view of cluster 1 GRNs. (D) Frontal and sagittal view of cluster 2 GRNs. (E) Frontal and sagittal view of cluster 3 GRNs. (F) Frontal and sagittal view of cluster 4 GRNs. (G) Frontal and sagittal view of cluster 5 GRNs. (H) Frontal and sagittal view of cluster 6 GRNs. (I) Frontal and sagittal view of cluster 7 GRNs. 


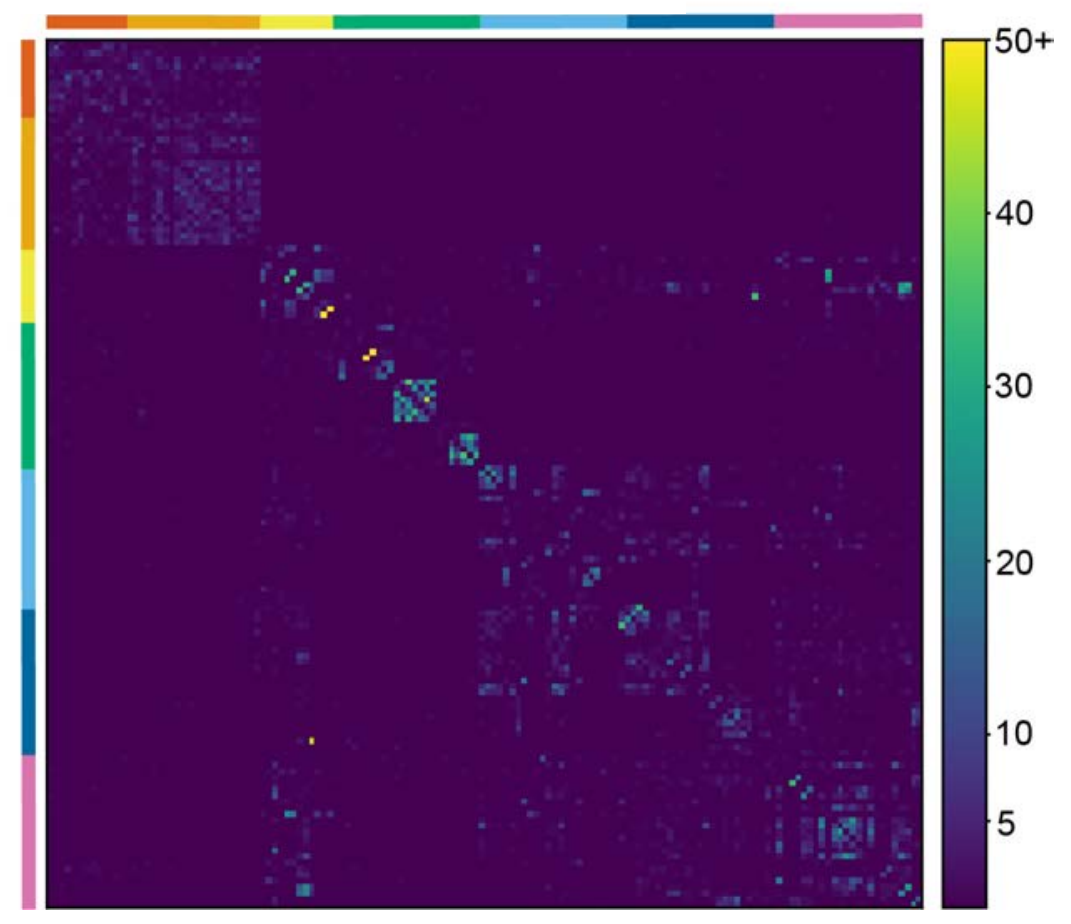

Figure 2 - figure supplement 2. Synaptic connections between GRNs.

Connectivity matrix of all GRNs. Presynaptic GRNs are arranged along the $y$ axis, postsynaptic GRNs along the $x$ axis. GRNs are ordered according to the purely morphological clustering tree shown in Figure 2.1 A. Cluster identities of GRNs are indicated by colored bars. Color coding within the matrix indicates the number of synapses from the pre- to the post-synaptic neuron as indicated in the legend. All synapses are included in this analysis. 


\section{Figure 3}

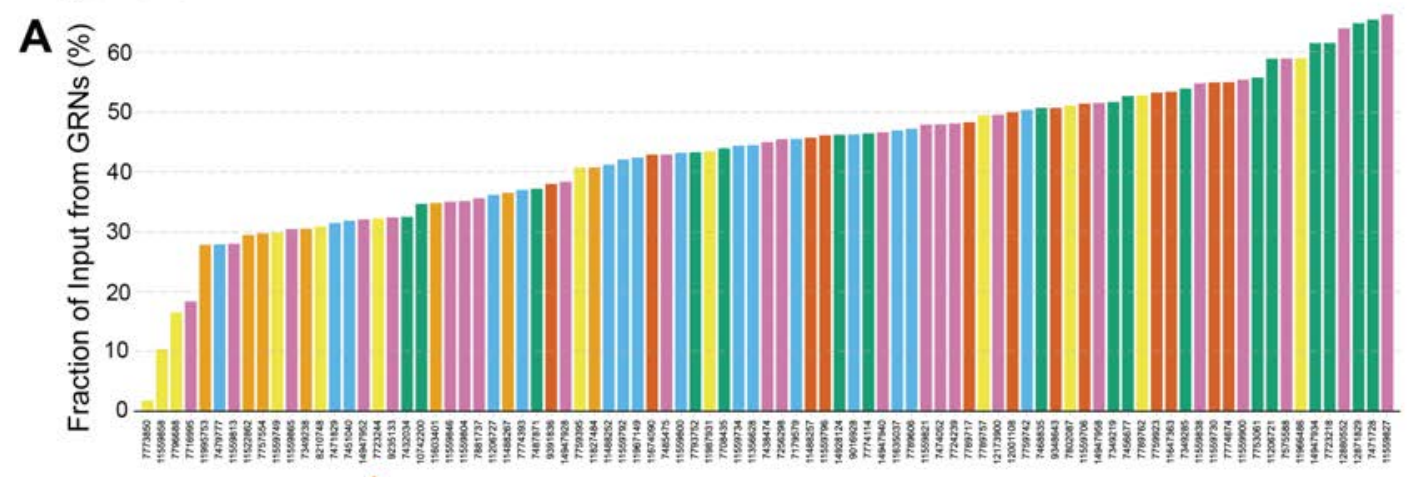

B
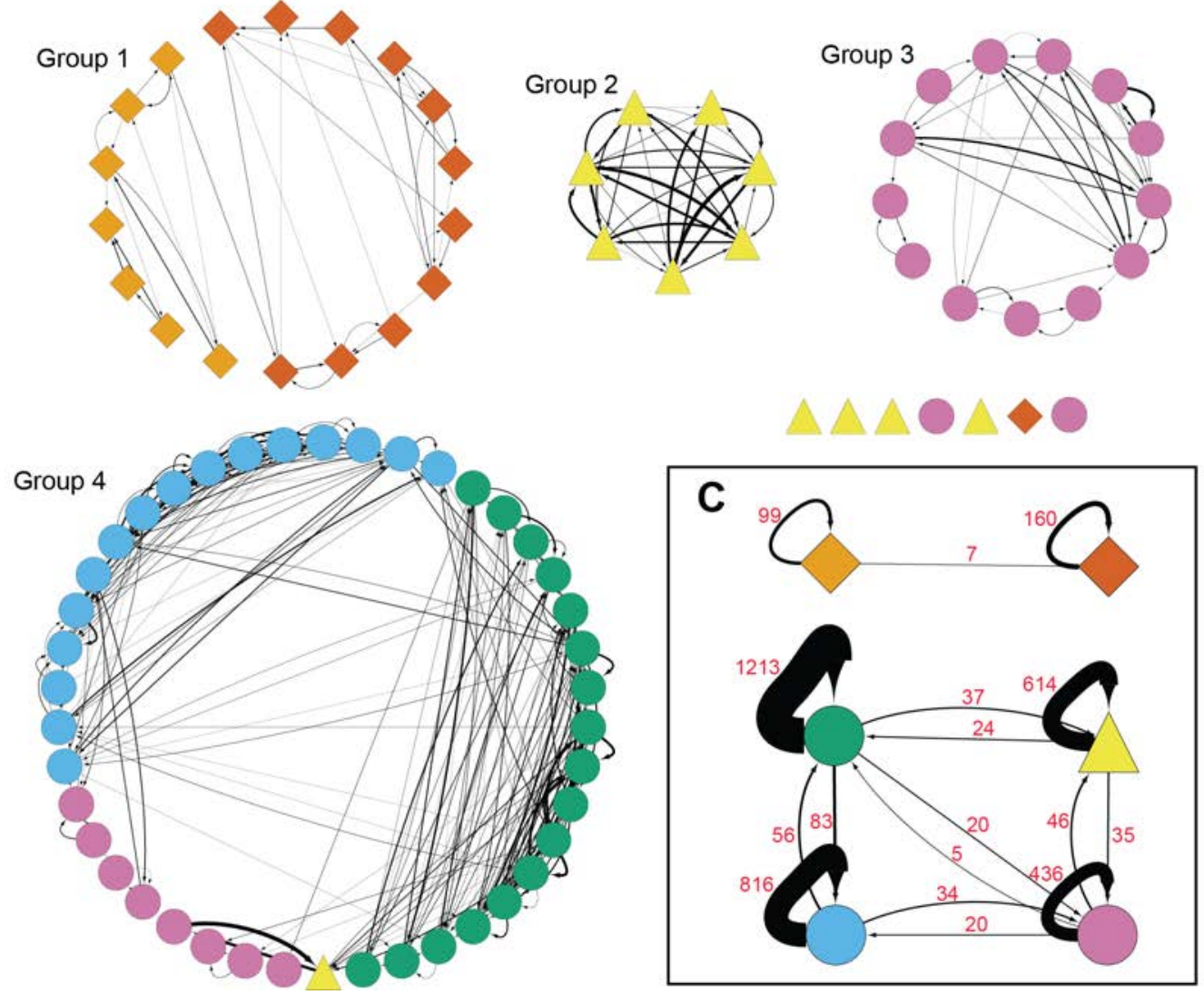

Figure 3. GRNs are highly interconnected via chemical synapses. (A) Percentage of GRN inputs to each GRN. Colors correspond to clusters established in Figure 2. (B) Connectivity between individual GRNs. Colors correspond to clusters established in Figure 2. Arrow thickness scales with the number of synapses comprising the connection. Only connections of at least 5 synapses are shown. (C) Connectivity between GRN clusters. Colors correspond to clusters established in Figure 2. Arrow thickness scales with the number of synapses comprising the connection, which is indicated in red. 
Figure 4

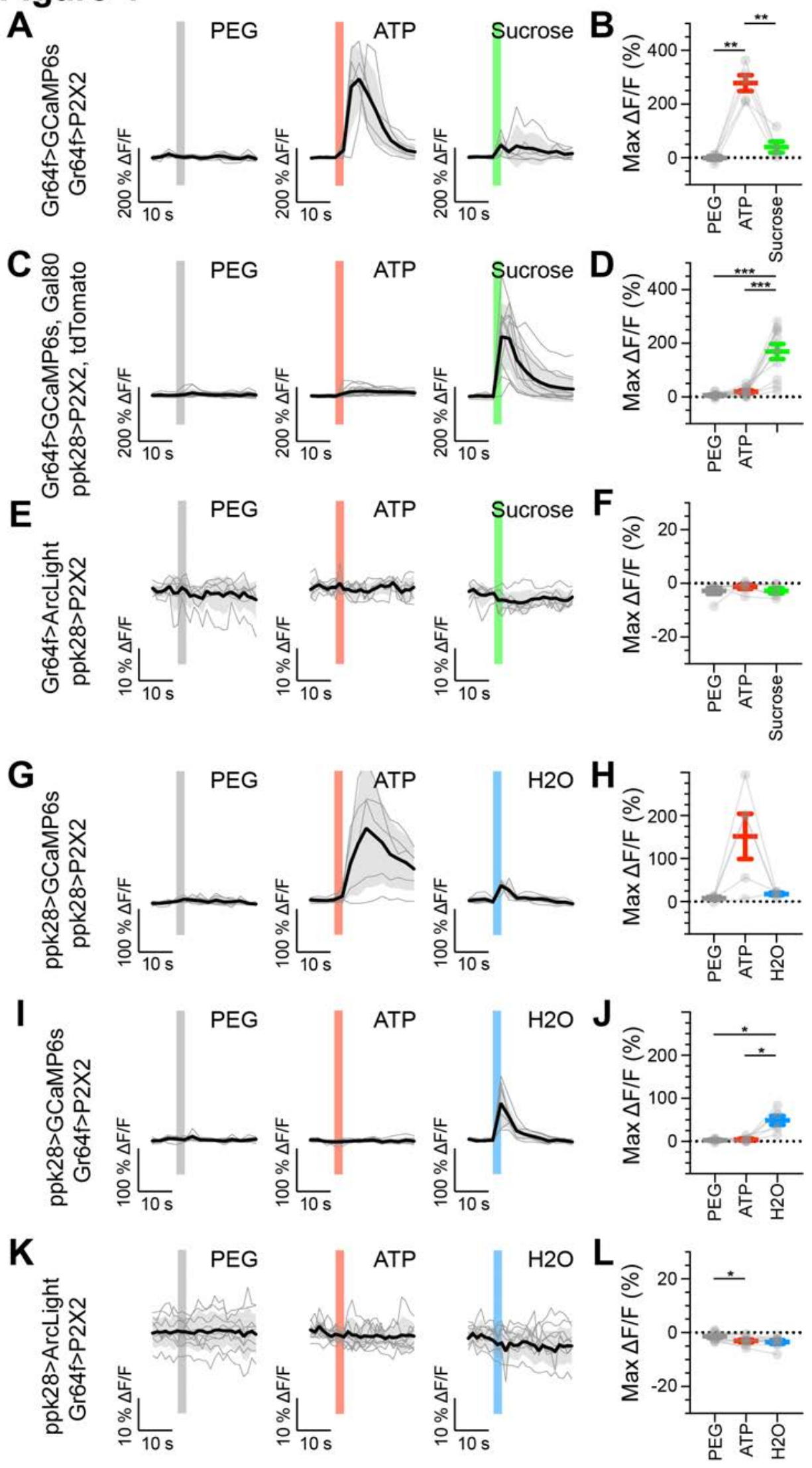


Figure 4. Sugar and water GRNs do not activate each other.

(A, B) Calcium responses of sugar GRNs expressing P2X2 and GCaMP6s to proboscis presentation of PEG as a negative control, ATP to activate P2X2, or sucrose as a positive control. GCaMP6s fluorescence traces $(\Delta F / F)(A)$ and maximum $\Delta F / F$ post stimulus presentation $(B), n=5$. Sugar GRNs responded to ATP, but the response to subsequent sucrose presentation was attenuated. (C, D) GCaMP6s responses of sugar GRNs in flies expressing P2X2 in water GRNs to PEG, ATP, and sucrose delivery, $\Delta F / F$ traces $(C)$ and maximum $\Delta F / F$ graph $(D), n=11$. ( $E, F)$ ArcLight responses of sugar GRNs in flies expressing $P 2 X 2$ in water $G R N s, \Delta F / F$ traces $(E)$ and maximum $\Delta F / F$ graph $(F), n=6 .(G, H)$ Calcium responses of water GRNs expressing P2X2 and GCaMP6s to proboscis delivery of PEG (negative control), ATP, and water (positive control), $\Delta F / F$ traces $(\mathrm{G})$ and maximum $\Delta F / F$ graph $(H), n=5$. Water $G R N$ s responded to ATP presentation, but the subsequent response to water was diminished. (I, J) GCaMP6s responses of water GRNs in flies expressing P2X2 in sugar GRNs to PEG, ATP, and water, $\Delta F / F$ traces $(\mathrm{I})$ and maximum $\Delta F / F$ graph $(J), n=6 .(K, L)$ ArcLight responses of water GRNs in flies expressing P2X2 in sugar GRNs to PEG, ATP, and water, $\Delta F / F$ traces $(\mathrm{K})$ and maximum $\Delta F / F$ graph $(\mathrm{L}), \mathrm{n}=9$.

For all traces, period of stimulus presentation is indicated by shaded bars. Traces of individual flies to the first of three taste stimulations (shown in Supplemental Figures 4.2, 4.3 and 4.7) are shown in grey, the average in black, with the SEM indicated by the grey shaded area. Repeated measures ANOVA with Tukey's multiple comparisons test, ${ }^{*} p<0.05,{ }^{* *} p<0.01,{ }^{* * *} p<0.001$. 


\section{Figure 4 - figure supplement 1}
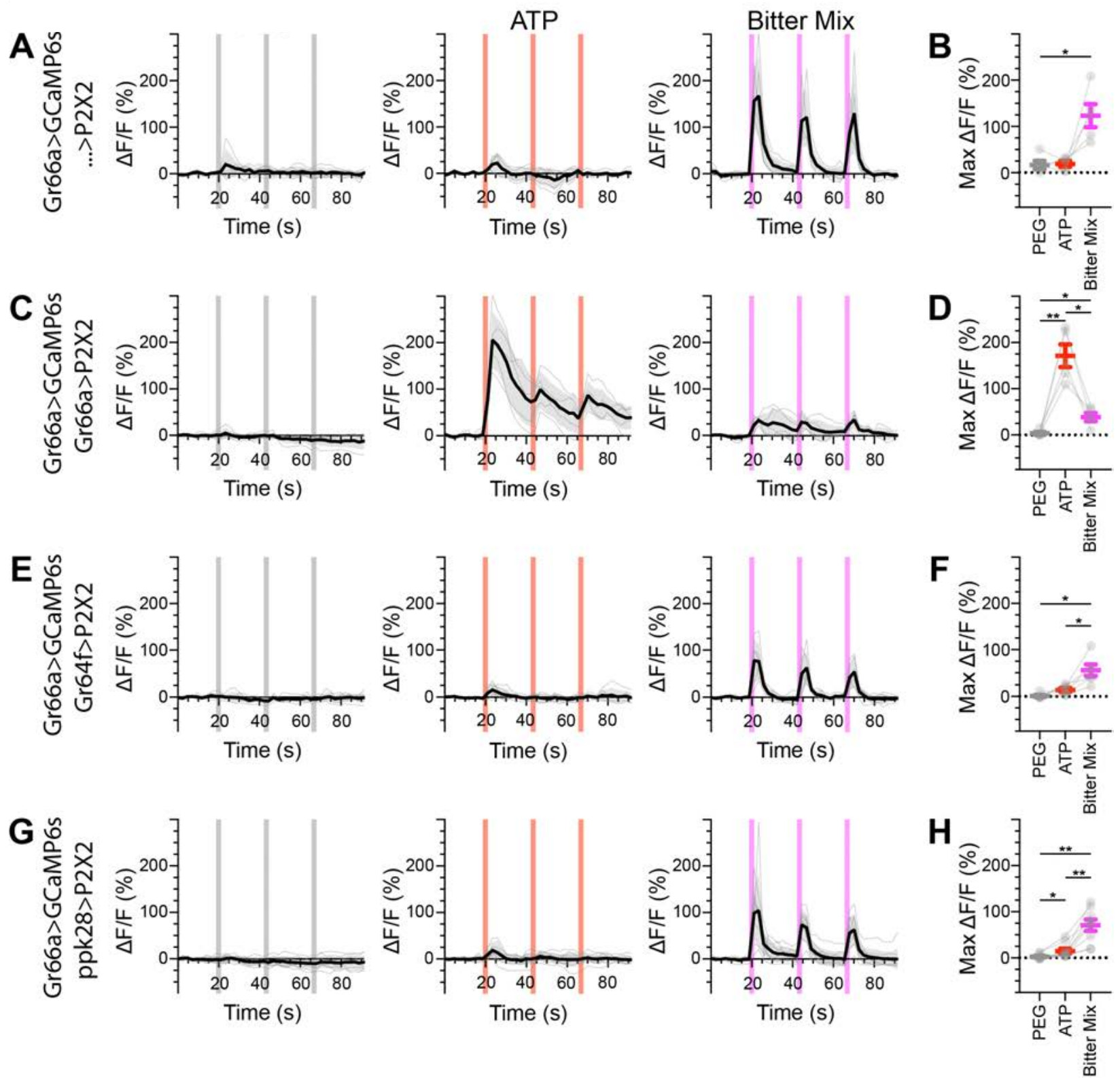
Figure 4 - figure supplement 1. Bitter GRNs do not respond to the activation of other GRN classes in fed flies.

(A, B) Calcium responses of bitter GRNs expressing GCaMP6s in a UAS-P2X2 background to proboscis presentation of PEG as a negative control, ATP, or a mixture of denatonium and caffeine, which are bitter compounds, as a positive control, GCaMP6s $\Delta F / F$ traces $(A)$ and maximum $\Delta F / F$ graph $(B), n=5$. (C, D) Calcium responses of bitter GRNs expressing GCaMP6s and P2X2 to PEG, ATP, or bitter delivery, $\Delta F / F$ traces $(C)$ and maximum $\Delta F / F$ graph $(D), n=5$. (E, F) GCaMP6s responses of bitter GRNs in flies expressing P2X2 in sugar GRNs to PEG, ATP, and bitter, $\Delta F / F$ traces $(E)$ and maximum $\Delta F / F$ graph $(F), n=6$. $(G, H)$ GCaMP6s responses of bitter GRNs in flies expressing P2X2 in water GRNs to delivery of PEG, ATP, or bitter to the proboscis, $\Delta F / F$ traces $(G)$ and maximum $\Delta F / F$ graph $(H), n=9$.

Period of stimulus presentation is indicated by shaded bars, 3 stimulations/fly. Traces of individual flies are shown in grey, the average in black, with the SEM indicated by the grey shaded area. Repeated measures ANOVA with Tukey's multiple comparisons test, ${ }^{*} p<0.05,{ }^{* *} p<0.01$. 
Figure 4 - figure supplement 2
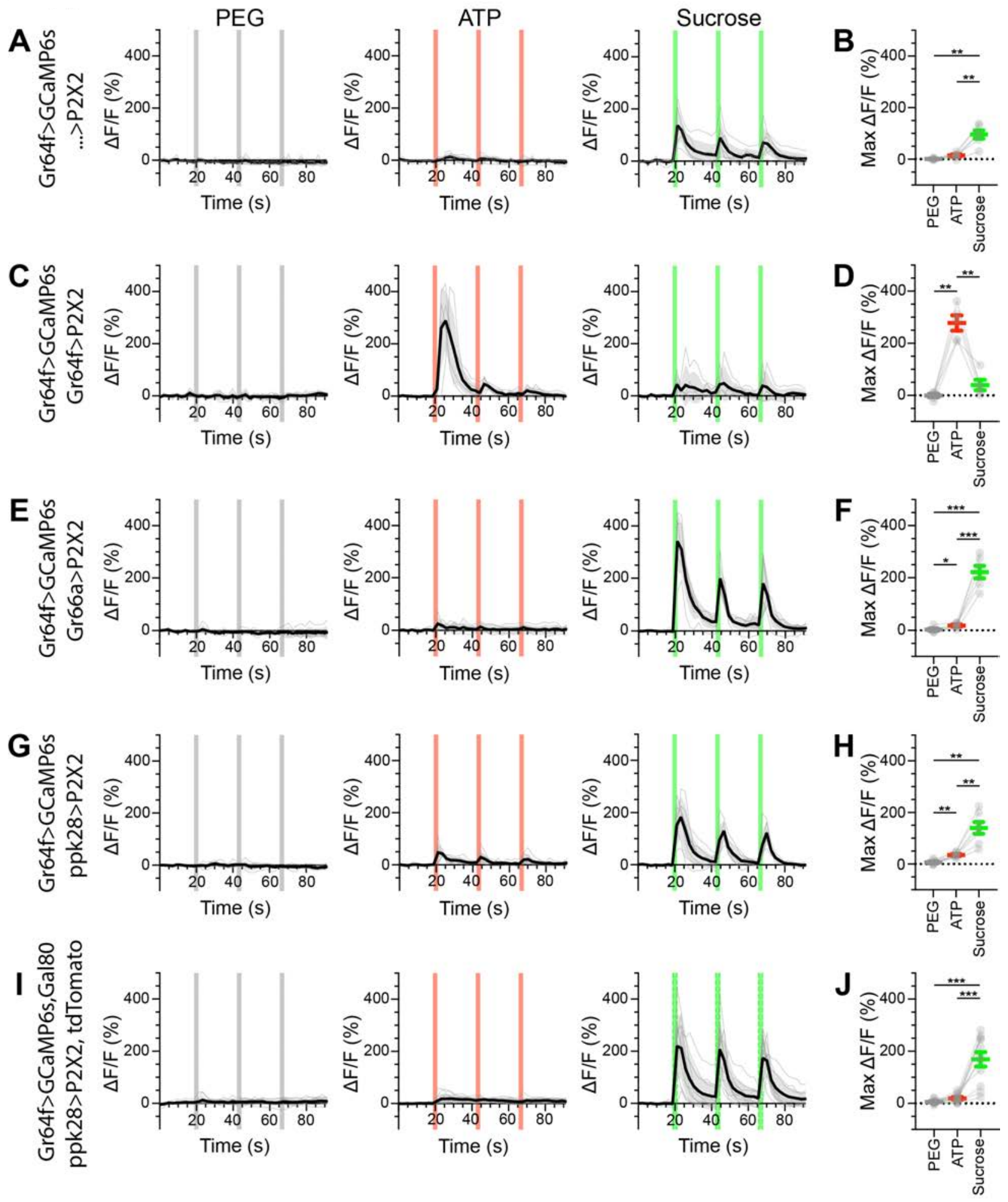
Figure 4 - figure supplement 2. Sugar GRNs do not respond to the activation of other GRN classes in fed flies.

(A, B) Calcium responses of sugar GRNs expressing GCaMP6s in a UAS-P2X2 background to proboscis presentation of PEG as a negative control, ATP, or sucrose as a positive control, GCaMP6s $\Delta \mathrm{F} / \mathrm{F}$ traces $(\mathrm{A})$ and maximum $\Delta \mathrm{F} / \mathrm{F}$ graph $(\mathrm{B}), \mathrm{n}=6$. (C, D) Calcium responses of sugar GRNs expressing GCaMP6s and P2X2 to PEG, ATP, or sucrose delivery, $\Delta F / F$ traces $(C)$ and maximum $\Delta F / F$ graph $(D), n=5 .(E, F)$ GCaMP6s responses of sugar GRNs in flies expressing P2X2 in bitter GRNs to PEG, ATP, and sucrose, $\Delta F / F$ traces $(E)$ and maximum $\Delta F / F$ graph $(F), n=6 .(G, H)$ GCaMP6s responses of sugar GRNs in flies expressing P2X2 in water GRNs to PEG, ATP, or sucrose presentation, $\Delta F / F$ traces $(G)$ and maximum $\Delta F / F$ graph $(H), n=7$. $(I, J)$ GCaMP6s responses of sugar GRNs in flies expressing P2X2 in water GRNs and Gal80 in sugar GRNs to inhibit P2X2 misexpression to PEG, ATP, or sucrose presentation, $\Delta F / F$ traces $(\mathrm{I})$ and maximum $\Delta F / F$ plots $(J), n=11$.

Period of stimulus presentation is indicated by shaded bars, 3 stimulations/fly. Data from first stimulation of $C$ and $K$ is shown in Figure 4A-D. Traces of individual flies are shown in grey, the average in black, with the SEM indicated by the grey shaded area. Repeated measures ANOVA with Tukey's multiple comparisons test ${ }^{*} p<0.05,{ }^{* *} p<0.01$, ${ }^{* * *} p<0.001$. 
Figure 4 - figure supplement 3
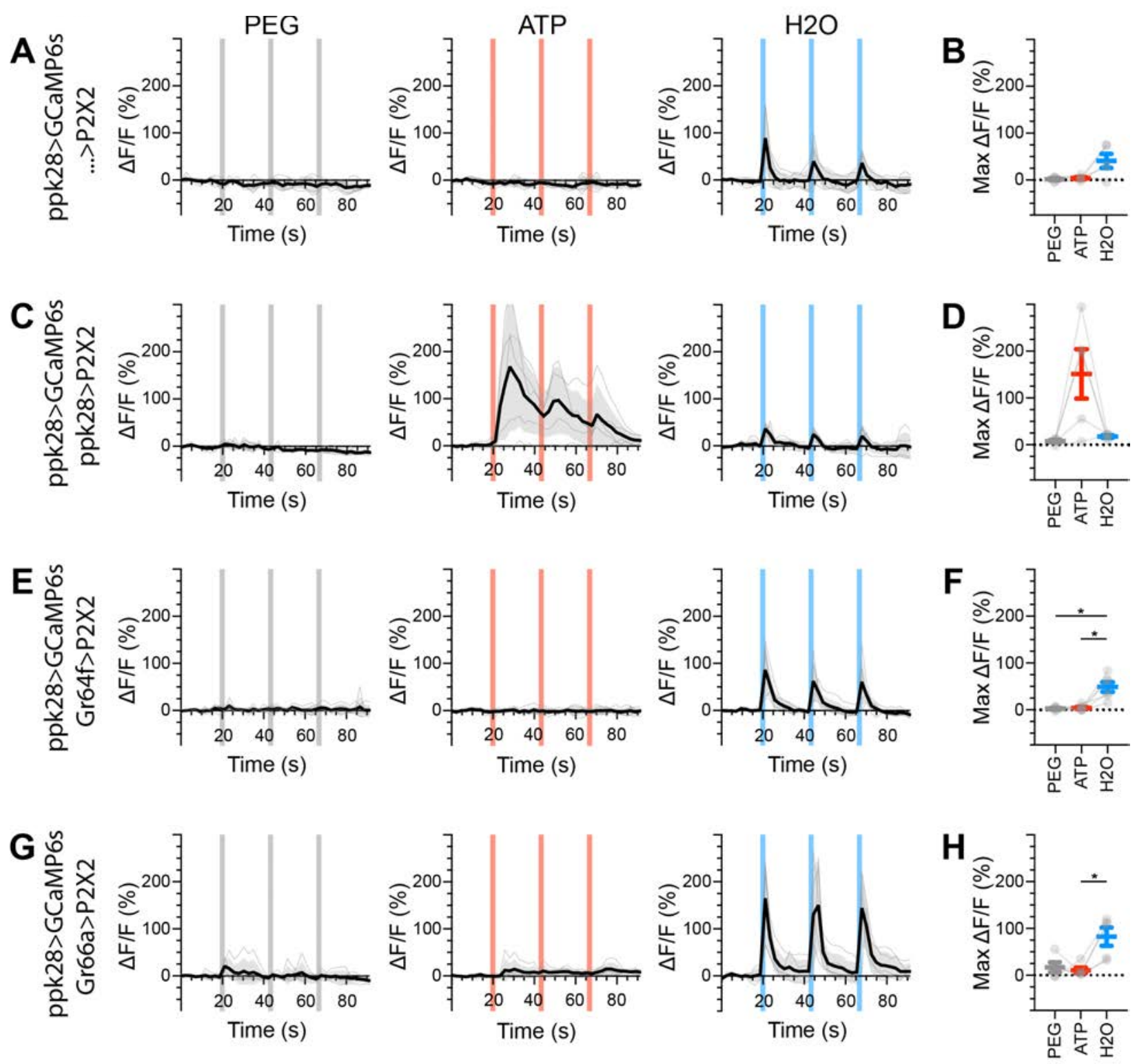
Figure 4 - figure supplement 3. Water GRNs do not respond to the activation of other GRN classes in fed flies.

(A, B) Calcium responses of water GRNs expressing GCaMP6s in a UAS-P2X2 background to proboscis presentation of PEG as a negative control, ATP, or water as a positive control, GCaMP6s $\Delta F / F$ traces $(A)$ and maximum $\Delta F / F$ graph $(B), n=5$. (C, D) Calcium responses of water GRNs expressing GCaMP6s and P2X2 to PEG, ATP, or water delivery, $\Delta F / F$ traces $(C)$ and maximum $\Delta F / F$ graph $(D), n=5$. $(E, F)$ GCaMP6s responses of water GRNs in flies expressing P2X2 in sugar GRNs to PEG, ATP, and water, $\Delta F / F$ traces $(E)$ and maximum $\Delta F / F$ graph $(F), n=6 .(G, H)$ GCaMP6s responses of water GRNs in flies expressing P2X2 in bitter GRNs upon PEG, ATP, or water presentation, $\Delta F / F$ traces $(G)$ and maximum $\Delta F / F$ graph $(H), n=5$. Period of stimulus presentation is indicated by shaded bars, 3 stimulations/fly. The first response in $\mathrm{C}$ and $\mathrm{E}$ is shown in Figure 4G-J. Traces of individual flies are shown in grey, the average in black, with the SEM indicated by the grey shaded area. Repeated measures ANOVA with Tukey's multiple comparisons test ${ }^{*} p<0.05$. 
Figure 4 - figure supplement 4
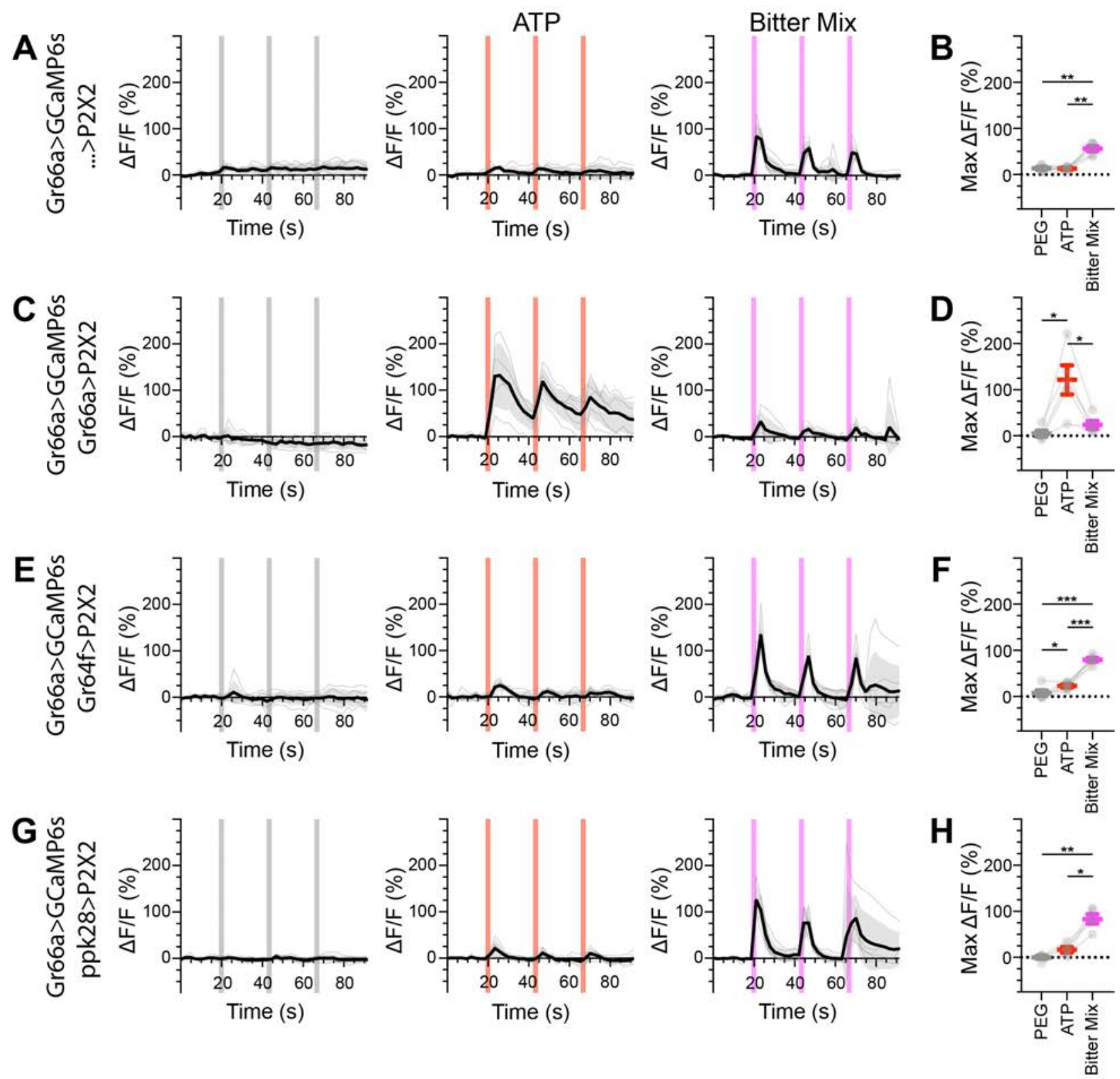
Figure 4 - figure supplement 4. Bitter GRNs do not respond to the activation of other GRN classes in food-deprived flies.

(A, B) Calcium responses of bitter GRNs expressing GCaMP6s in a UAS-P2X2 background to proboscis presentation of PEG as a negative control, ATP, or a mixture of the bitter compounds denatonium and caffeine as a positive control, GCaMP6s $\Delta F / F$ traces $(A)$ and maximum $\Delta F / F$ graph $(B), n=6$. $(C, D)$ Calcium responses of bitter GRNs expressing GCaMP6s and P2X2 to PEG, ATP, or bitter delivery, $\triangle F / F$ traces (C) and maximum $\Delta F / F$ graph $(D), n=5$. $(E, F)$ GCaMP6s responses of bitter GRNs in flies expressing $P 2 X 2$ in sugar GRNs to PEG, ATP, and bitter, $\triangle F / F$ traces $(E)$ and maximum $\Delta F / F$ graph $(F), n=6 .(G, H)$ GCaMP6s responses of bitter GRNs in flies expressing $P 2 X 2$ in water GRNs to delivery of PEG, ATP, or bitter, $\triangle F / F$ traces $(G)$ and maximum $\Delta \mathrm{F} / \mathrm{F}$ graph $(\mathrm{H}), \mathrm{n}=5$.

Period of stimulus presentation is indicated by shaded bars, 3 stimulations/fly. Flies were food-deprived for 23-26 hours. Traces of individual flies are shown in grey, the average in black, with the SEM indicated by the grey shaded area. Repeated measures ANOVA with Tukey's multiple comparisons test, ${ }^{*} p<0.05,{ }^{* *} p<0.01,{ }^{* * *} p<0.001$. 
Figure 4 - figure supplement 5
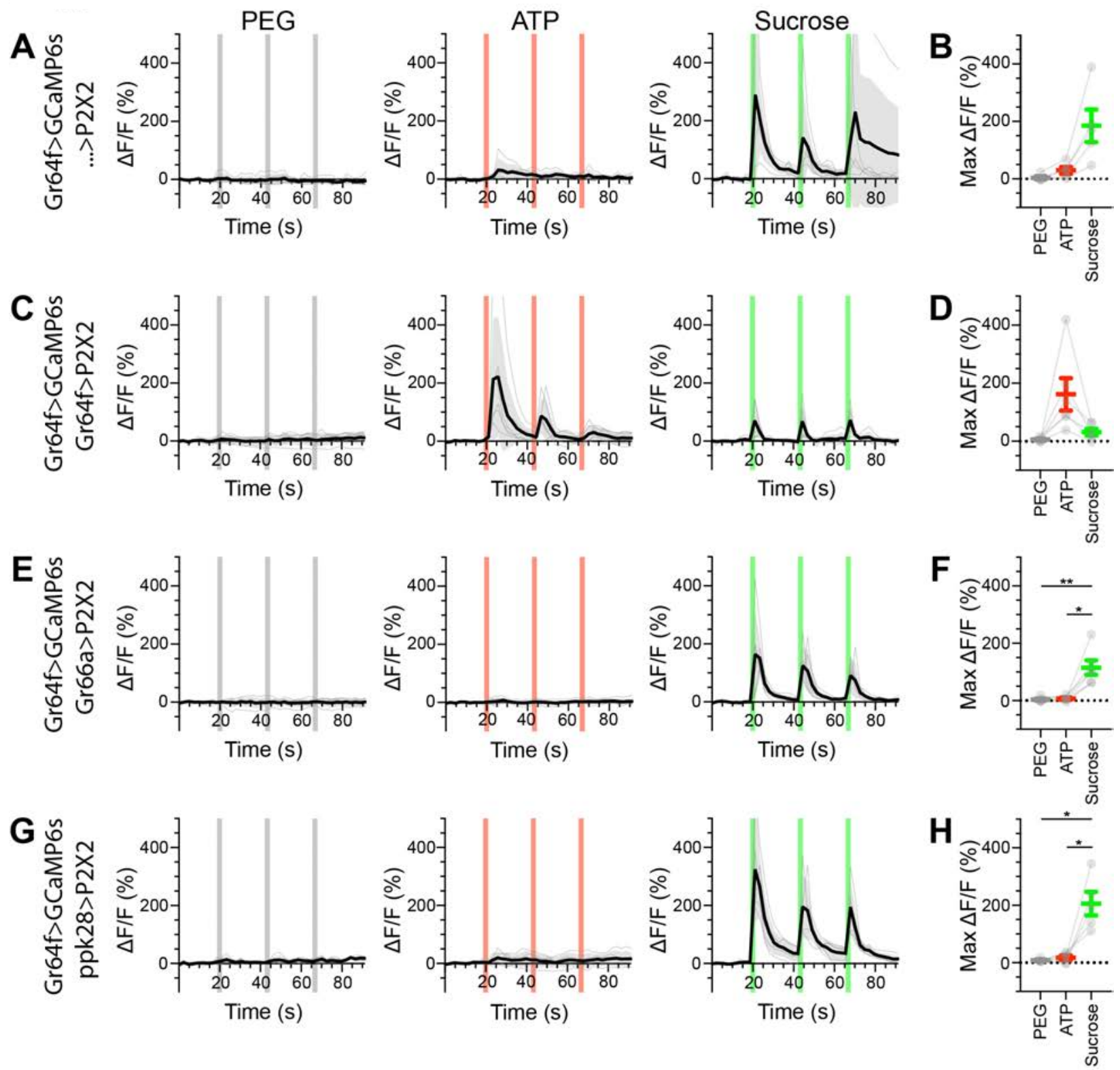
Figure 4 - figure supplement 5. Sugar GRNs do not respond to the activation of other GRN classes in food-deprived flies.

(A, B) Calcium responses of sugar GRNs expressing GCaMP6s in a UAS-P2X2 background to proboscis presentation of PEG as a negative control, ATP, or sucrose as a positive control, GCaMP6s $\Delta \mathrm{F} / \mathrm{F}$ traces $(\mathrm{A})$ and maximum $\Delta \mathrm{F} / \mathrm{F}$ graph $(\mathrm{B}), \mathrm{n}=5$. $(\mathrm{C}$, D) Calcium responses of sugar GRNs expressing GCaMP6s and P2X2 to PEG, ATP, or sucrose delivery, $\Delta F / F$ traces $(C)$ and maximum $\Delta F / F$ graph $(D), n=6$. $(E, F)$ GCaMP6s responses of sugar GRNs in flies expressing P2X2 in bitter GRNs to PEG, ATP, and sucrose, $\Delta F / F$ traces $(E)$ and maximum $\Delta F / F$ graph $(F), n=6 .(G, H)$ GCaMP6s responses of sugar GRNs in flies expressing P2X2 in water GRNs to PEG, ATP, and sucrose presentation to the proboscis, $\Delta F / F$ traces $(G)$ and maximum $\Delta F / F$ graph $(H), n$ $=5$.

Period of stimulus presentation is indicated by shaded bars, 3 stimulations/fly. Flies were food-deprived for 23-26 hours. Traces of individual flies are shown in grey, the average in black, with the SEM indicated by the grey shaded area. Repeated measures ANOVA with Tukey's multiple comparisons test ${ }^{*} p<0.05,{ }^{* *} p<0.01$. 
Figure 4 - figure supplement 6
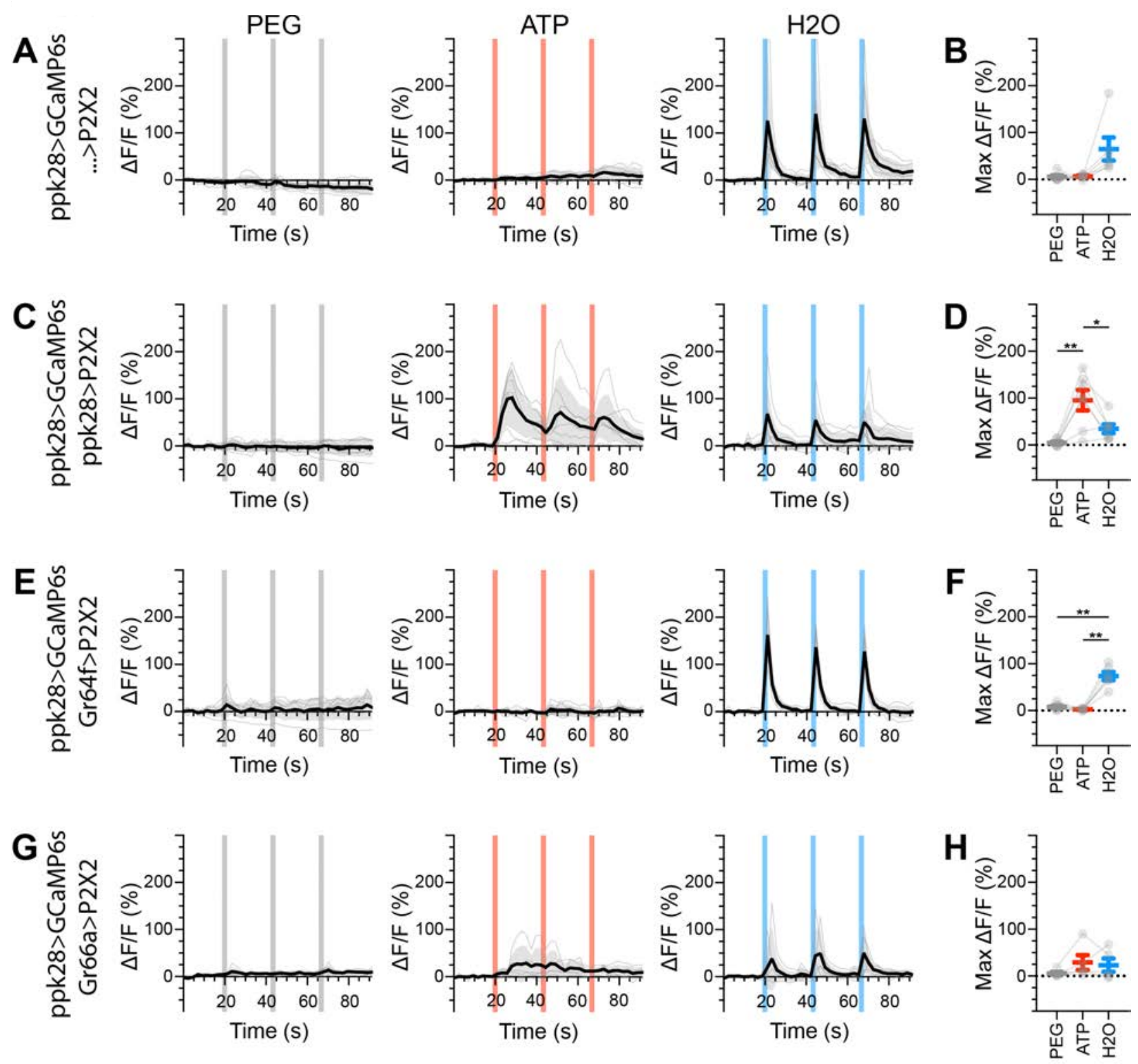
Figure 4 - figure supplement 6. Water GRNs do not respond to the activation of other GRN classes in food-deprived flies.

(A, B) Calcium responses of water GRNs expressing GCaMP6s in a UAS-P2X2 background to proboscis presentation of PEG as a negative control, ATP, or water as a positive control, GCaMP6s $\Delta F / F$ traces $(A)$ and maximum $\Delta F / F$ graph $(B), n=6$. (C, D) Calcium responses of water GRNs expressing GCaMP6s and P2X2 to PEG, ATP, or water delivery, $\Delta F / F$ traces $(C)$ and maximum $\Delta F / F$ graph $(D), n=7$. $(E, F)$ GCaMP6s responses of water GRNs in flies expressing P2X2 in sugar GRNs to PEG, ATP, and water, $\Delta F / F$ traces $(E)$ and maximum $\Delta F / F$ graph $(F), n=6 .(G, H)$ GCaMP6s responses of water GRNs in flies expressing P2X2 in bitter GRNs to PEG, ATP, and water delivery, $\Delta F / F$ traces $(G)$ and maximum $\Delta F / F$ graph $(H), n=5$.

Period of stimulus presentation is indicated by shaded bars, 3 stimulations/fly. Flies were food-deprived for 23-26 hours. Traces of individual flies are shown in grey, the average in black, with the SEM indicated by the grey shaded area. Repeated measures ANOVA with Tukey's multiple comparisons test ${ }^{*} p<0.05,{ }^{* *} p<0.01$. 
Figure 4 - figure supplement 7
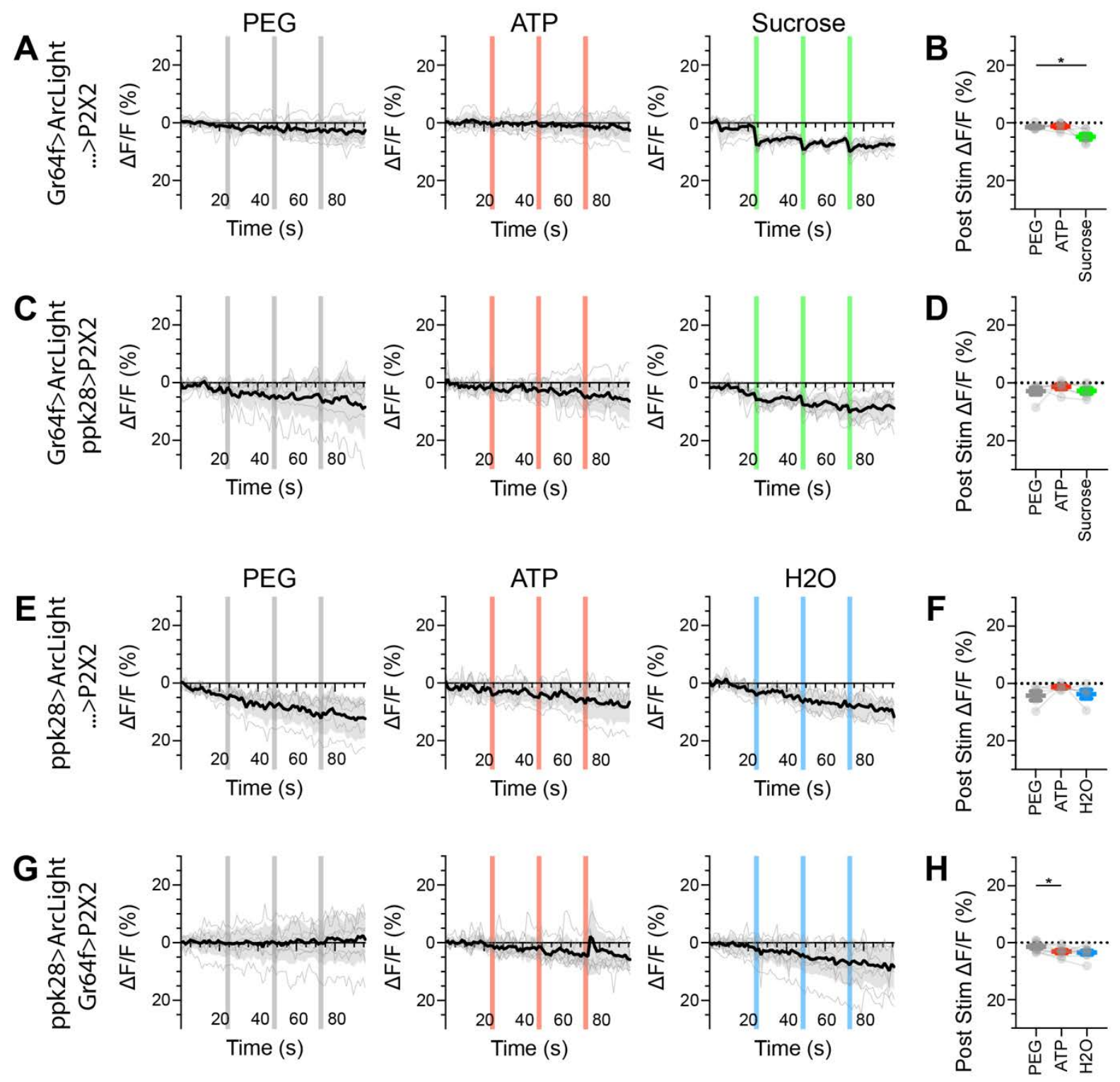
Figure 4 - figure supplement 7. Sugar and water GRNs do not show voltage responses upon reciprocal activation.

(A, B) ArcLight responses of sugar GRNs in a UAS-P2X2 background to proboscis presentation of PEG as a negative control, ATP, or sucrose as a positive control. ArcLight fluorescence traces $(\Delta F / F)(A)$ and maximum $\Delta F / F$ post stimulus presentation $(B), n=6 .(C, D)$ ArcLight responses of sugar GRNs in flies expressing P2X2 in water GRNs to PEG, ATP, and sucrose delivery, $\Delta F / F$ traces $(C)$ and maximum $\Delta F / F$ graph (D), $n=6 .(E, F)$ ArcLight responses of water GRNs in a UAS-P2X2 background to proboscis delivery of PEG, ATP, and water (positive control), $\triangle F / F$ traces $(E)$ and maximum $\Delta F / F$ graph $(F), n=5$. $(\mathrm{G}, \mathrm{H})$ ArcLight responses of water $\mathrm{GRNs}$ in flies expressing $P 2 X 2$ in sugar GRNs to PEG, ATP, and water delivery, $\Delta F / F$ traces $(G)$ and maximum $\Delta F / F$ graph $(H), n=9$.

Period of stimulus presentation is indicated by shaded bars, 3 stimulations/fly. The first response in $C$ and $G$ is shown in Figure 2-4 E, F, K, L. Traces of individual flies to three taste stimulations are shown in grey, the average in black, with the SEM indicated by the grey shaded area. Repeated measures ANOVA with Tukey's multiple comparisons test, ${ }^{*} p<0.05$. 
bioRxiv preprint doi: https://doi.org/10.1101/2021.12.08.471796; this version posted December 9, 2021. The copyright holder for this preprint (which was not certified by peer review) is the author/funder, who has granted bioRxiv a license to display the preprint in perpetuity. It is made available under aCC-BY-NC-ND 4.0 International license.

Figure 5
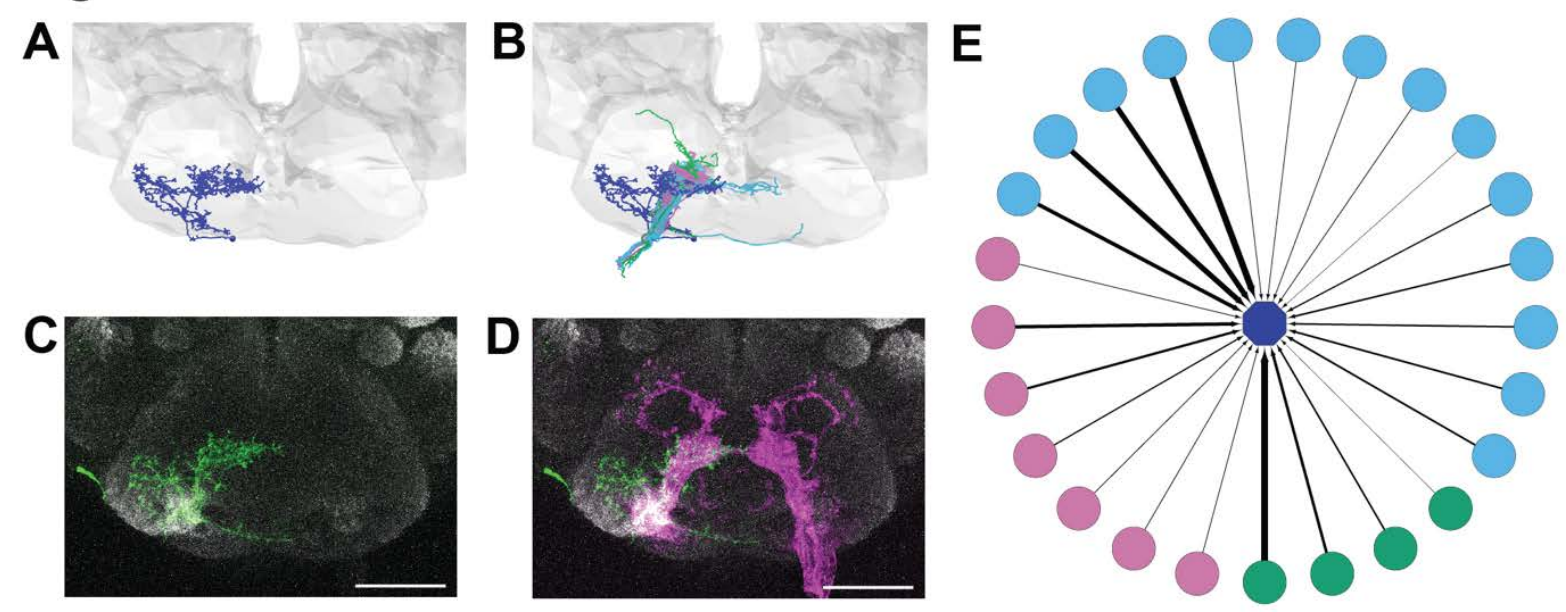

$\mathbf{F}$

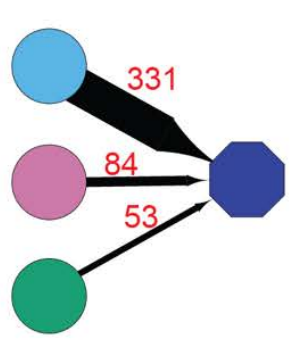

G

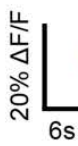

H

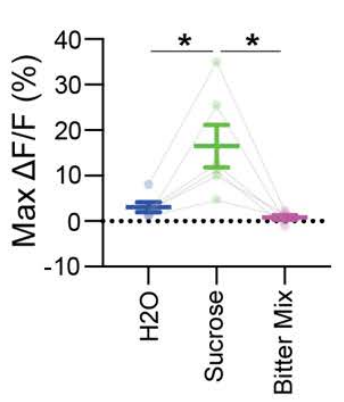

I

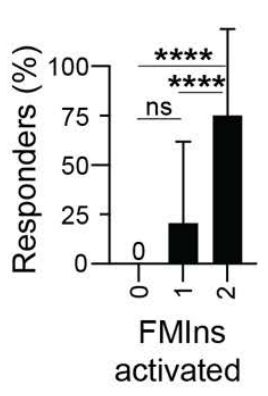


Figure 5. A second order gustatory neuron downstream of sugar GRNs in the EM responds selectively to sugar presentation.

(A) Reconstruction of FMIn in the FAFB dataset (B) FMIn and its GRN inputs in the EM dataset. GRN colors correspond to clusters established in Figure 2. (C) Light level image of a single FMIn generated by flippase 1026A mediated mosaicism from GmR81E10-Gal4 (scale bar $=25 \mu \mathrm{m}$ ) (D) FMIn (green) overlaps with sugar taste projections (magenta) in light-level imagery (scale bar $=25 \mu \mathrm{m}$ ). (E) GRN inputs to FMIn identified in the EM. Colors correspond to clusters established in Figure 2. Arrow thickness scales with the number of synapses comprising the connection. Only connections of at least 5 synapses are shown. (F) GRN inputs to FMln by GRN cluster. Colors correspond to clusters established in Figure 2. Arrow thickness scales with the number of synapses comprising the connection, which is indicated in red. Only connections of at least 5 synapses are considered. (G-H) GCaMP7s responses of FMIn cell bodies to tastant presentations to the proboscis. $(G)$ The responses of flies presented with water (blue), sucrose (green), and a bitter mixture of denatonium and caffeine (pink). Individual traces are shown in thin lines colored corresponding to tastant identity, averages in thicker lines with the SEM indicated by shaded areas. $(H)$ Maximum $\Delta F / F$ graph. $n=6$, Repeated measures ANOVA with Tukey's multiple comparisons test ${ }^{*} p<0.5$. (G) Fraction of flies performing PER to light with the number of FMIns activated using ReaChR. 0 FMIn: $n=9 ; 1$ FMIn: $n=29 ; 2$ FMIns: $n=36$. Unpaired t test, ${ }^{* * * *} p<0.0001$. 

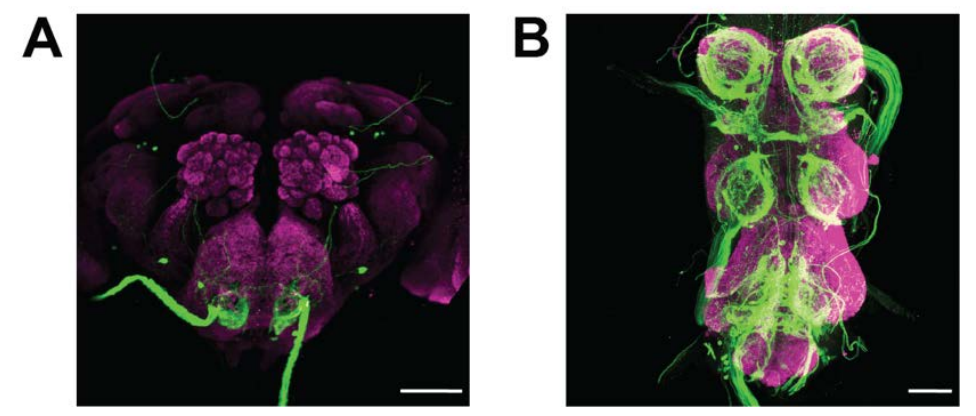

Figure 5 - figure supplement 1. Expression pattern of GmR81E10-Gal4 in the central nervous system.

(A) Expression pattern of GmR81E10-Gal4 in the brain (scale bar $=50 \mu \mathrm{m})(B)$

Expression pattern of GmR81E10-Gal4 in the ventral nerve cord (scale bar $=50 \mu \mathrm{m}$ ). 


\section{References}

Bates AS, Manton JD, Jagannathan SR, Costa M, Schlegel P, et al. 2020. The natverse, a versatile toolbox for combining and analysing neuroanatomical data. Elife 9

Bogovic JA, Otsuna H, Heinrich L, Ito M, Jeter J, et al. 2020. An unbiased template of the Drosophila brain and ventral nerve cord. PLoS One 15: e0236495

Braun E, Geurten B, Egelhaaf M. 2010. Identifying prototypical components in behaviour using clustering algorithms. PLoS One 5: e9361

Buhmann J, Sheridan A, Malin-Mayor C, Schlegel P, Gerhard S, et al. 2021. Automatic detection of synaptic partners in a whole-brain Drosophila electron microscopy data set. Nat Methods 18: 771-74

Cameron P, Hiroi M, Ngai J, Scott K. 2010. The molecular basis for water taste in Drosophila. Nature 465: 91-5

Cao G, Platisa J, Pieribone VA, Raccuglia D, Kunst M, Nitabach MN. 2013. Genetically targeted optical electrophysiology in intact neural circuits. Cell 154: 904-13

Chen TW, Wardill TJ, Sun Y, Pulver SR, Renninger SL, et al. 2013. Ultrasensitive fluorescent proteins for imaging neuronal activity. Nature 499: 295-300

Chen Z, Wang Q, Wang Z. 2010. The amiloride-sensitive epithelial Na+ channel PPK28 is essential for drosophila gustatory water reception. J Neurosci 30: 6247-52

Costa M, Manton JD, Ostrovsky AD, Prohaska S, Jefferis GS. 2016. NBLAST: Rapid, Sensitive Comparison of Neuronal Structure and Construction of Neuron Family Databases. Neuron 91: 293-311

Dahanukar A, Foster K, van der Goes van Naters WM, Carlson JR. 2001. A Gr receptor is required for response to the sugar trehalose in taste neurons of Drosophila. Nat Neurosci 4: 1182-6

Dahanukar A, Lei YT, Kwon JY, Carlson JR. 2007. Two Gr genes underlie sugar reception in Drosophila. Neuron 56: 503-16

Dana H, Sun Y, Mohar B, Hulse BK, Kerlin AM, et al. 2019. High-performance calcium sensors for imaging activity in neuronal populations and microcompartments. Nat Methods 16: 649-657

Gordon MD, Scott K. 2009. Motor control in a Drosophila taste circuit. Neuron 61: 37384

Hampel S, Eichler K, Yamada D, Bock DD, Kamikouchi A, Seeds AM. 2020. Distinct subpopulations of mechanosensory chordotonal organ neurons elicit grooming of the fruit fly antennae. Elife 9

Harris DT, Kallman BR, Mullaney BC, Scott K. 2015. Representations of Taste Modality in the Drosophila Brain. Neuron 86: 1449-60

Hartenstein V, Omoto JJ, Ngo KT, Wong D, Kuert PA, et al. 2018. Structure and development of the subesophageal zone of the Drosophila brain. I. Segmental architecture, compartmentalization, and lineage anatomy. J Comp Neurol 526: 632

Horne JA, Langille C, McLin S, Wiederman M, Lu Z, et al. 2018. A resource for the Drosophila antennal lobe provided by the connectome of glomerulus VA1v. Elife 7 
Huang P, Sahai-Hernandez P, Bohm RA, Welch WP, Zhang B, Nystul T. 2014. Enhancer-trap flippase lines for clonal analysis in the Drosophila ovary. G3 (Bethesda) 4: 1693-9

Inagaki HK, Jung Y, Hoopfer ED, Wong AM, Mishra N, et al. 2014. Optogenetic control of Drosophila using a red-shifted channelrhodopsin reveals experiencedependent influences on courtship. Nat Methods 11: 325-32

Jaeger AH, Stanley M, Weiss ZF, Musso PY, Chan RC, et al. 2018. A complex peripheral code for salt taste in Drosophila. Elife 7

Jefferis GS, Potter CJ, Chan AM, Marin EC, Rohlfing T, et al. 2007. Comprehensive maps of Drosophila higher olfactory centers: spatially segregated fruit and pheromone representation. Cell 128: 1187-203

Jenett A, Rubin GM, Ngo TT, Shepherd D, Murphy C, et al. 2012. A GAL4-driver line resource for Drosophila neurobiology. Cell Rep 2: 991-1001

Jourjine N, Mullaney BC, Mann K, Scott K. 2016. Coupled Sensing of Hunger and Thirst Signals Balances Sugar and Water Consumption. Cell 166: 855-66

Kwon JY, Dahanukar A, Weiss LA, Carlson JR. 2011. Molecular and cellular organization of the taste system in the Drosophila larva. J Neurosci 31: 15300-9

Lee T, Luo L. 1999. Mosaic Analysis with a Repressible Cell Marker for Studies of Gene Function in Neuronal Morphogenesis. Neuron 22: 451-61

Li F, Lindsey JW, Marin EC, Otto N, Dreher M, et al. 2020a. The connectome of the adult Drosophila mushroom body provides insights into function. Elife 9

Li PH, Lindsey LF, Januszewski M, Tyka M, Maitin-Shepard J, et al. 2019. Automated Reconstruction of a Serial-Section EM Drosophila Brain with Flood-Filling Networks and Local Realignment. Microscopy and Microanalysis 25: 1364-65

Lima SQ, Miesenbock G. 2005. Remote control of behavior through genetically targeted photostimulation of neurons. Cell 121: 141-52

Miroschnikow A, Schlegel P, Schoofs A, Hueckesfeld S, Li F, et al. 2018. Convergence of monosynaptic and polysynaptic sensory paths onto common motor outputs in a Drosophila feeding connectome. Elife 7

Miyamoto T, Slone J, Song X, Amrein H. 2012. A fructose receptor functions as a nutrient sensor in the Drosophila brain. Cell 151: 1113-25

Miyazaki T, Ito K. 2010. Neural architecture of the primary gustatory center of Drosophila melanogaster visualized with GAL4 and LexA enhancer-trap systems. J Comp Neurol 518: 4147-81

Nayak S, Singh R. 1983. Sensilla on the tarsal segments and mouthparts of adult Drosophila melanogaster meigen Int J Insect Morphol \& Embryol 12: 273-91

Nern A, Pfeiffer BD, Svoboda K, Rubin GM. 2011. Multiple new site-specific recombinases for use in manipulating animal genomes. Proc Natl Acad Sci U S A 108: 14198-203

Otsana H, Ito M, Kawase T. 2018. Color depth MIP mask search: a new tool to expedite Split-GAL4 creation. bioRxiv. https://doi.org/10.1101/318006

Rajashekhar KP, Singh R. 1994. Neuroarchitecture of the Tritocerebrum of Drosophila melanogaster. J Comp Neurol 349: 633-45

Saalfeld S, Cardona A, Hartenstein V, Tomancak P. 2009. CATMAID: collaborative annotation toolkit for massive amounts of image data. Bioinformatics 25: 1984-6 
Schindelin J, Arganda-Carreras I, Frise E, Kaynig V, Longair M, et al. 2012. Fiji: an open-source platform for biological-image analysis. Nat Methods 9: 676-82

Schlegel P, Bates AS, Sturner T, Jagannathan SR, Drummond N, et al. 2021. Information flow, cell types and stereotypy in a full olfactory connectome. Elife 10

Schneider-Mizell CM, Gerhard S, Longair M, Kazimiers T, Li F, et al. 2016. Quantitative neuroanatomy for connectomics in Drosophila. Elife 5

Scott K, Brady R, Cravchik A, Morozov P, Rzhetsky A, et al. 2001. A Chemosensory Gene Family Encoding Candidate Gustatory and Olfactory Receptors in Drosophila Cell 104: 661-73

Shannon P, Markiel A, Ozier O, Baliga NS, Wang JT, et al. 2003. Cytoscape: a software environment for integrated models of biomolecular interaction networks. Genome Res 13: 2498-504

Sterne GR, Otsuna H, Dickson BJ, Scott K. 2021. Classification and genetic targeting of cell types in the primary taste and premotor center of the adult Drosophila brain. Elife 10

Stocker RF. 1994. The Organization of the Chemosensory System in Drosophila melanogaster: a Review Cell Tissue Res 275: 3-26

Takemura SY, Bharioke A, Lu Z, Nern A, Vitaladevuni S, et al. 2013. A visual motion detection circuit suggested by Drosophila connectomics. Nature 500: 175-81

Takemura SY, Xu CS, Lu Z, Rivlin PK, Parag T, et al. 2015. Synaptic circuits and their variations within different columns in the visual system of Drosophila. Proc Natl Acad Sci U S A 112: 13711-6

Thistle R, Cameron P, Ghorayshi A, Dennison L, Scott K. 2012. Contact chemoreceptors mediate male-male repulsion and male-female attraction during Drosophila courtship. Cell 149: 1140-51

Thorne N, Chromey C, Bray S, Amrein H. 2004. Taste perception and coding in Drosophila. Curr Biol 14: 1065-79

Tobin WF, Wilson RI, Lee WA. 2017. Wiring variations that enable and constrain neural computation in a sensory microcircuit. Elife 6

Wang JW, Wong AM, Flores J, Vosshall LB, Axel R. 2003. Two-Photon Calcium Imaging Reveals an Odor-Evoked Map of Activity in the Fly Brain. Cell 112: 27182

Wang Z, Singhvi A, Kong P, Scott K. 2004. Taste representations in the Drosophila brain. Cell 117: 981-91

Weiss LA, Dahanukar A, Kwon JY, Banerjee D, Carlson JR. 2011. The molecular and cellular basis of bitter taste in Drosophila. Neuron 69: 258-72

Yao Z, Macara AM, Lelito KR, Minosyan TY, Shafer OT. 2012. Analysis of functional neuronal connectivity in the Drosophila brain. J Neurophysiol 108: 684-96

Zheng Z, Lauritzen JS, Perlman E, Robinson CG, Nichols M, et al. 2018. A Complete Electron Microscopy Volume of the Brain of Adult Drosophila melanogaster. Cell 174: $730-43$ e22 